SHEP 99-09

\title{
A gauge invariant exact renormalization group I
}

\author{
Tim R. Morris \\ Department of Physics, University of Southampton, \\ Highfield, Southampton SO17 1BJ, UK
}

\begin{abstract}
A manifestly gauge invariant continuous renormalization group flow equation is constructed for pure $S U(N)$ gauge theory. The formulation makes sense without gauge fixing and manifestly gauge invariant calculations may thus be carried out. The flow equation is naturally expressed in terms of fluctuating Wilson loops, with the effective action appearing as an integral over a 'gas' of Wilson loops. At infinite $N$, the effective action collapses to a path integral over the trajectory of a single particle describing one Wilson loop. We show that further regularization of these flow equations is needed. (This is introduced in part II.)
\end{abstract}

hep-th/9910058

July, 1999. 


\section{Introduction and motivation}

Our main motivation for the present work is to obtain an elegant gauge invariant Wilsonian renormalization group [1] framework formulated directly in the continuum, as a first step for non-perturbative analytic approximation methods. Quite generally such methods can prove powerful, 1 and of course there is a clear need for a better non-perturbative understanding of gauge theory. However, several other issues are naturally resolved in the process of solving this first step.

In recent years there has been substantial progress in solving supersymmetric gauge theories [7]. These methods involve computing a low energy gauge invariant Wilsonian effective action, which however, because of the lack of a suitable framework, is never precisely defined. Whilst we concentrate here solely on pure Yang-Mills theory, we see no essential difficulty in generalising the flow equations to include fermions and scalars and indeed spacetime supersymmetry. It is clear then that our framework can underpin these ideas [7] [8].

Our framework is here formulated for an $S U(N)$ gauge group, and is a concise exposition of that reported in ref. [3] (see also [9]). All of the ideas presented here adapt to other gauge groups, with minor alterations.2

Whilst it is an interesting academic issue to establish the existence of a gauge invariant Wilsonian effective action and corresponding flow equation, its use would ultimately be limited without powerful non-perturbative approximation schemes. Fortunately, a beautiful approximation scheme lies waiting to be developed: namely the large $N$ limit where the gauge group is e.g. $S U(N)$ [10]-[14]. Typically, the starting point for these methods has been Dyson-Schwinger equations for Wilson loops derived at the bare level. Progress has been hampered by the lack of corresponding renormalised equations [13]. One of the most attractive features of the exact renormalization group $(\mathrm{RG})$ is the fact that solutions may readily be expressed directly in renormalised terms [4]. Thus combining these two approaches removes one obstacle to solving the large $N$ limit.

We will see that the large $N$ limit of the Wilson flow equations however results in an intriguing picture which is (somehow) dual to the Dyson-Schwinger approach. For example, in this picture the gauge fields appear not integrated over but take the rôle of background

1 See for example the reviews 2 2 -6$]$.

2 arising from adapting the completeness relation for the generators 
field 'spectators' while the Wilson loop, which is fixed (but selectable) in the DysonSchwinger approach, is now dynamical and integrated over. The analogue of Migdal's observation [1] that the large $N$ limit may be expressed in terms of the expectation value of a single Wilson loop 3 is here reflected in the fact that the continuum limit of the Wilsonian effective action, which at finite $N$ may be written in terms of (infinite) sums of integrals of products of Wilson loops, at infinite $N$ reduces to a single integral over configurations of just one Wilson loop. The flow equations reduce to equations determining the path integral measure for this Wilson loop. Operators, which may be viewed as perturbations of this action, also take the form of averages over Wilson loop configurations. These are nothing but the continuum counterparts of the 'interpolating' operators used in lattice gauge theory to create propagating glueball states and study their wavefunctions. This picture is thus ideally suited to describing both the gauge fields and the low energy (e.g. bound state) degrees of freedom.

Let us emphasise that the solution of the large $N$ limit in this way, collapses the quantum field theory to a form of single particle quantum mechanics. By far the most exciting possibility raised by this viewpoint, in our opinion, is that it may finally open the door to the solution of large $N$ gauge theory. (Quite apart from the obvious theoretical attractions, the large $N$ limit is expected to be accurate in practical situations e.g. $10 \%$ accuracy is expected for many quantities in $S U(3)$ Yang-Mills [15].) In the present paper, this picture lies just below the surface. We will leave to future work a fuller investigation of this description, and the simplifications at large $N$ that ensue. Nevertheless, this picture was central in guiding us to the construction of a consistent flow equation.

A particular problem that has to be faced in this direction, is that a gauge invariant effective cutoff function, similarly to gauge invariant higher derivative regularisation, is not sufficient to regulate all ultra-violet divergences. One loop divergences slip through [16]. We cure this problem in the sequel by adapting the equations to a novel spontaneously broken supersymmetric gauge theory, in which the heavy partners play the rôle of PauliVillars regulator fields [9]. In this way all the attractive aspects of the present framework are preserved while curing this one remaining problem.

The most attractive feature is surely the property that gauge invariance is explicitly maintained at all stages (no gauge fixing or BRST ghosts are required), resulting in elegant and highly constrained relations. One important consequence is that there is no

3 due to correlators of Wilson loops decoupling in the planar limit [10] 
wavefunction renormalization. The only quantity requiring renormalization is the coupling constant!

Actually, honest non-perturbative approaches to non-Abelian gauge theory that proceed by gauge fixing, must face up to the challenging problem of Gribov copies [17]. Here, these problems are entirely avoided.

In previous exact RG approaches to gauge theory the authors have gauge fixed, and also allowed the effective cutoff to break the gauge invariance. They then seek to recover it in the limit that the cutoff is removed [18] [19]. As we have indicated, the present development follows a very different route. In sec. 2, we review Polchinski's form of Wilson's exact RG molding it into a form suitable for such a generalisation. In sec. 3, we then generalise this to $U(1)$ gauge fields as a stepping stone to the full non-Abelian generalisation. We introduce here several important properties of these generalisations, namely that gauge invariance may be exactly preserved, that solutions may be found without gauge fixing, and that nontrivial generalisations exist which leave the partition function invariant and continue to correspond to integrating out. In sec. 4, we introduce the full non-Abelian generalisation, demonstrate that the gauge field cannot renormalize, introduce the concept of a 'wine', coincident line identities, the definition of the coupling $g$, and discuss some qualitative criteria satisfied by the formulation namely, 'quasilocality', 'ultralocality', and 'integrating out'. In sec. 5, we develop the gauge field expansion in terms of traces and products of traces, introducing the effective vertices, and demonstrate that the large $N$ limit of the effective action has only a single trace. In sec. 6, we introduce and develop the Wilson loop representation which we deliberately leave this late in the paper to emphasise that the formalism stands separately from this interpretation, although we believe this enables a powerful intuition. The large $N$ limit of the effective action is then seen to correspond to a form of quantum mechanics for a single particle and the flow equation is seen to determine the measure over the fluctuating Wilson loops. In sec. 7, we use this interpretation to rapidly develop some of the general properties of the vertices: the trivial Ward identities expressing exact preservation of gauge invariance, charge conjugation invariance, Lorentz invariance, and the coincident line identities. Sec. 8 sets out the perturbative expansion, demonstrating that the Wilson loop diagrams are also Feynman diagrams. In this section, we solve for the classical two, three and four point functions, along the way showing explicitly how manifestly gauge invariant perturbative computations may be performed, and explaining how the resulting formulae are highly constrained by gauge invariance considerations, in fact to the underlying Wilson loop picture. We then explain how the 
$\beta$ function is determined and give the relevant one-loop contribution. We analyse this and show how this is at least quadratically divergent (in four dimensions) whatever the choice of cutoff function. This causes subtleties with gauge invariance due to momentum integral surface terms that do not vanish. We also show that the coefficients of some of the divergences are not even polynomial in the momenta. Appendix A provides a large momentum analysis of the vertices, necessary to draw these conclusions. Along the way we demonstrate that this bad ultraviolet behaviour is a necessary consequence of the exact preservation of gauge invariance (and briefly discuss the colinear and small momentum regimes which are even more highly constrained by gauge invariance). Nevertheless we will see in part II [9], that these ultraviolet problems can be cured, as already indicated above.

\section{The Polchinski equation}

We work in $D$ Euclidean dimensions. For two functions $f(x)$ and $g(y)$ and a momentum space kernel $W\left(p^{2} / \Lambda^{2}\right)$, where $\Lambda$ is the effective cutoff, we introduce the shorthand:

$$
\begin{aligned}
f \cdot W \cdot g & :=\iint d^{D} x d^{D} y f(x) W_{x y} g(y) \\
\text { where } \quad W_{x y} & \equiv \int \frac{d^{D} p}{(2 \pi)^{D}} W\left(p^{2} / \Lambda^{2}\right) \mathrm{e}^{i p .(x-y)} .
\end{aligned}
$$

Polchinski's [20] version of Wilson's exact RG [1], for the effective interaction of a scalar field $S^{i n t}[\varphi]$, may then be written

$$
\Lambda \frac{\partial}{\partial \Lambda} S^{i n t}=-\frac{1}{\Lambda^{2}} \frac{\delta S^{i n t}}{\delta \varphi} \cdot c^{\prime} \cdot \frac{\delta S^{i n t}}{\delta \varphi}+\frac{1}{\Lambda^{2}} \frac{\delta}{\delta \varphi} \cdot c^{\prime} \cdot \frac{\delta S^{i n t}}{\delta \varphi}
$$

Here $c\left(p^{2} / \Lambda^{2}\right)>0$ is a smooth, i.e. infinitely differentiable, ultra-violet cutoff profile, and prime denotes differentiation with respect to its argument. The cutoff, which modifies propagators $1 / p^{2}$ to $c / p^{2}$, satisfies $c(0)=1$ so that low energies are unaltered, and $c\left(p^{2} / \Lambda^{2}\right) \rightarrow 0$ as $p^{2} / \Lambda^{2} \rightarrow \infty$ sufficiently fast that all Feynman diagrams are ultraviolet regulated. We may write the regularised kinetic term (i.e. the Gaussian fixed point) as

$$
\hat{S}=\frac{1}{2} \partial_{\mu} \varphi \cdot c^{-1} \cdot \partial_{\mu} \varphi
$$

In terms of the total effective action $S[\varphi]=\hat{S}+S^{i n t}$, and $\Sigma_{1}:=S-2 \hat{S}$, the exact RG equation reads

$$
\Lambda \frac{\partial}{\partial \Lambda} S=-\frac{1}{\Lambda^{2}} \frac{\delta S}{\delta \varphi} \cdot c^{\prime} \cdot \frac{\delta \Sigma_{1}}{\delta \varphi}+\frac{1}{\Lambda^{2}} \frac{\delta}{\delta \varphi} \cdot c^{\prime} \cdot \frac{\delta \Sigma_{1}}{\delta \varphi}
$$


(up to a vacuum energy term that was discarded in (2.2) [20]). The flow in $S$ may be shown directly to correspond to integrating out higher energy modes [1] [4] [21]- 24], while leaving the partition function $\mathcal{Z}=\int \mathcal{D} \varphi \mathrm{e}^{-S}$ invariant. (For our purposes we may absorb all source terms into $S$ as spacetime dependent couplings.) Indeed the invariance of $\mathcal{Z}$ follows from (2.4) because

$$
\Lambda \frac{\partial}{\partial \Lambda} \mathrm{e}^{-S}=-\frac{1}{\Lambda^{2}} \frac{\delta}{\delta \varphi} \cdot c^{\prime} \cdot\left(\frac{\delta \Sigma_{1}}{\delta \varphi} \mathrm{e}^{-S}\right)
$$

is a total functional derivative.

\section{A flow equation for Abelian gauge fields}

We motivate the form of our non-Abelian exact RG by first developing an Abelian version. For $U(1)$ gauge theory, we write the covariant derivative as $D_{\mu}=\partial_{\mu}-i g A_{\mu}$, where $g$ is the coupling. Clearly if we replace the Gaussian fixed point solution (2.3) by the gauge invariant

$$
\hat{S}=\frac{1}{4} F_{\mu \nu} \cdot c^{-1} \cdot F_{\mu \nu}
$$

(where $F_{\mu \nu}=\partial_{\mu} A_{\nu}-\partial_{\nu} A_{\mu}$ ), then the flow equation

$$
\Lambda \frac{\partial}{\partial \Lambda} S=-\frac{1}{\Lambda^{2}} \frac{\delta S}{\delta A_{\mu}} \cdot c^{\prime} \cdot \frac{\delta \Sigma_{1}}{\delta A_{\mu}}+\frac{1}{\Lambda^{2}} \frac{\delta}{\delta A_{\mu}} \cdot c^{\prime} \cdot \frac{\delta \Sigma_{1}}{\delta A_{\mu}}
$$

is also gauge invariant, while still preserving the invariance of the partition function under the flow (by (2.5), replacing $\varphi$ by $A_{\mu}$ ). Furthermore, by utilising ref. [25] this may readily be shown to correspond directly to integrating out higher energy modes [3].

Note that no gauge fixing has taken place, nor is it necessary for finding solutions to (3.2). This exact preservation of gauge invariance will have the important consequence, when generalised to non-Abelian gauge theory, that $D_{\mu}$ cannot renormalise. It is thus convenient to redefine $D_{\mu}=\partial_{\mu}-i A_{\mu}$, by changing variables $A_{\mu} \mapsto A_{\mu} / g$, since $A_{\mu}$ will then not suffer wavefunction renormalisation and only the coupling $g$ will renormalise. If we also redefine $\hat{S} \mapsto \hat{S} / g^{2}$ (i.e. keep definition (3.1) for the new fields), (3.2) becomes

$$
\Lambda \frac{\partial}{\partial \Lambda} S+\frac{\beta}{g} \int d^{D} x A_{\mu}(x) \frac{\delta S}{\delta A_{\mu}(x)}=-\frac{1}{\Lambda^{2}} \frac{\delta S}{\delta A_{\mu}} \cdot c^{\prime} \cdot \frac{\delta \Sigma_{g}}{\delta A_{\mu}}+\frac{1}{\Lambda^{2}} \frac{\delta}{\delta A_{\mu}} \cdot c^{\prime} \cdot \frac{\delta \Sigma_{g}}{\delta A_{\mu}},
$$

where $\beta:=\Lambda \partial g / \partial \Lambda$, and $\Sigma_{g}:=g^{2} S-2 \hat{S}$. Of course it is still the case that $\Lambda \frac{\partial}{\partial \Lambda} \mathrm{e}^{-S}$ is a total functional derivative (after addition of a vacuum energy term, the Jacobian for $\left.A_{\mu} \mapsto A_{\mu} / g\right)$. 
This RG equation is not yet in a convenient form for generalisation to non-Abelian gauge theory, because the new ' $\beta$ ' term on the left of (3.3) is not manifestly gauge invariant. 6 We now come to a central observation of the paper. There are many other flow equations which have the property that $\Lambda \frac{\partial}{\partial \Lambda} \mathrm{e}^{-S}$ is a total functional derivative, and thus leave

$$
\mathcal{Z}=\int \mathcal{D} A \mathrm{e}^{-S}
$$

invariant. Furthermore a subset also correspond to integrating out higher energy modes, even though direct derivations [1] [21]-[24] may no longer be possible (cf. ref.[3] and the next section). In particular, here we can simply drop this annoying ' $\beta$ ' term!

Pure $U(1)$ gauge theory is not a good testing ground for these assertions because the only continuum solution is $S=\hat{S} / g^{2}$ with $\beta=0$, i.e. the Gaussian fixed point of free photons. It was thus an instructive exercise for us to perform a similar analysis for (2.4) and $\lambda \varphi^{4}$ theory, and demonstrate explicitly that universal terms, e.g. the one-loop $\beta$ function $(D=4)$, are unchanged after mapping $\varphi \mapsto \varphi / \lambda^{1 / 4}$ and dropping the ' $\beta$ ' term, and indeed to check that this is true even on more baroque alterations. Since the nonAbelian gauge theory exact RG corresponds to our most important such example, we will not report further on these exercises.

To summarise, for $U(1)$ gauge theory we may write the exact RG as

$$
\begin{aligned}
\Lambda \frac{\partial}{\partial \Lambda} S & =-\frac{1}{\Lambda^{2}} \frac{\delta S}{\delta A_{\mu}} \cdot c^{\prime} \cdot \frac{\delta \Sigma_{g}}{\delta A_{\mu}}+\frac{1}{\Lambda^{2}} \frac{\delta}{\delta A_{\mu}} \cdot c^{\prime} \cdot \frac{\delta \Sigma_{g}}{\delta A_{\mu}}, \\
\text { where } \quad \Sigma_{g} & =g^{2} S-2 \hat{S},
\end{aligned}
$$

and $\hat{S}$ is given by (3.1). This leaves the partition function (3.4) invariant:

$$
\Lambda \frac{\partial}{\partial \Lambda} \mathrm{e}^{-S}=-\frac{1}{\Lambda^{2}} \frac{\delta}{\delta A_{\mu}} \cdot c^{\prime} \cdot\left(\frac{\delta \Sigma_{g}}{\delta A_{\mu}} \mathrm{e}^{-S}\right)
$$

In the next section, we explain why it also corresponds to integrating out higher energy modes [3].

\footnotetext{
4 Gauge invariance follows only once we assume $S$ is gauge invariant, i.e. here $\partial_{\mu}\left(\delta S / \delta A_{\mu}\right)=0$.
} In the non-Abelian case gauge invariance would not hold separately for each order in $\hbar$. 


\section{A flow equation for non-Abelian gauge fields}

We work with the gauge group $S U(N)$. (These ideas may easily be extended to general gauge groups). We are interested in formulating an exact RG for the gauge field $A_{\mu}(x)=$ $A_{\mu}^{a}(x) \tau^{a}$, the connection for the covariant derivative $D_{\mu}=\partial_{\mu}-i A_{\mu}$. The generators $\left(\tau^{a}\right)^{i}{ }_{j}$ are taken to be Hermitian, in the fundamental representation, and orthonormalised as $\operatorname{tr}\left(\tau^{a} \tau^{b}\right)=\frac{1}{2} \delta^{a b}$.

Gauge transformations are of the form $\delta A_{\mu}=D_{\mu} \cdot \omega:=\left[D_{\mu}, \omega\right]$ where $\omega(x)=\omega^{a}(x) \tau^{a}$. Let us stress that exact preservation of this relationship immediately implies that $A_{\mu}$ cannot run (and thus has the naïve unit scaling dimension): if the gauge field were to suffer multiplicative wavefunction renormalization by $Z$, we would have to write $A_{\mu} \mapsto A_{\mu} / Z$, destroying the gauge invariance since then $\delta A_{\mu}=(Z-1) \partial_{\mu} \omega+D_{\mu} \cdot \omega$. This argument fails in the gauge fixed theory only because $\omega$ is replaced by a ghost field in the BRS transformation [26] leading to pointwise products of fields ( $\sim A_{\mu} \times$ ghost) which are themselves ill defined without further renormalization.

The field strength is $F_{\mu \nu}:=i\left[D_{\mu}, D_{\nu}\right]$. It will be useful to define

$$
\frac{\delta}{\delta A_{\mu}(x)}:=2 \tau^{a} \frac{\delta}{\delta A_{\mu}^{a}}
$$

This transforms homogeneously, and its properties can be understood as follows. To avoid the momentarily extraneous aspects - the $x$ dependence and $\mu$ index - let $A^{a}$ be an adjoint representation and write analogously, $\partial / \partial A=2 \tau^{a} \partial / \partial A^{a}$. If $s(A)$ is a function of $A$ such that

$$
\delta s(A)=\operatorname{tr} \delta A Y,
$$

then by the completeness relation for $S U(N)$, we effectively isolate $Y$ :

$$
\frac{\partial s}{\partial A}=Y-\frac{1}{N} \operatorname{tr} Y
$$

This leads, up to $O(1 / N)$, to 'sowing'

$$
\operatorname{tr} X \frac{\partial s}{\partial A}=\operatorname{tr} X Y-\frac{1}{N} \operatorname{tr} X \operatorname{tr} Y
$$

and 'splitting'

$$
\delta X=Y \delta A Z \quad \Longrightarrow \quad \operatorname{tr} \frac{\partial}{\partial A} X=\operatorname{tr} Y \operatorname{tr} Z-\frac{1}{N} \operatorname{tr} Y Z
$$


Given a kernel $W\left(p^{2} / \Lambda^{2}\right)$, we construct ${ }_{x l}^{i}\{W\}_{j y}^{k}$, the 'wine' [3], a functional of the kernel which is its gauge covariantization, incorporating parallel transport of the tensor representation. Thus, if $v_{k}^{j}(y)$ and $u_{i}^{l}(x)$ are two $N \otimes \bar{N}$ representations of the gauge group $S U(N)$, the gauge invariant generalisation of (2.1) is

$$
u\{W\} v:=\int d^{D} x d^{D} y u_{i}^{l}(x){ }_{x l}^{i}\{W\}_{j y}^{k} v_{k}^{j}(y)
$$

where without loss of generality we may insist that $\{W\}$ satisfies $u\{W\} v \equiv v\{W\} u$. The index flow, or parallel transport, is illustrated in fig. 1.

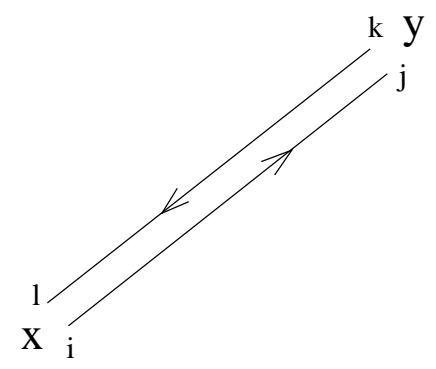

Fig.1. Index flow in eqn.(4.6).

Expanding this in terms of the gauge field $A_{\mu}$ defines the wine-vertices:

$$
\begin{array}{r}
{ }_{x l}^{i}\{W\}_{j y}^{k}= \\
\sum_{m, n=0}^{\infty} \int d^{D} x_{1} \cdots d^{D} x_{n} d^{D} y_{1} \cdots d^{D} y_{m} W_{\mu_{1} \cdots \mu_{n}, \nu_{1} \cdots \nu_{m}}\left(x_{1}, \cdots, x_{n} ; y_{1}, \cdots, y_{m} ; x, y\right) \\
{\left[A_{\mu_{1}}\left(x_{1}\right) \cdots A_{\mu_{n}}\left(x_{n}\right)\right]_{j}^{i}\left[A_{\nu_{1}}\left(y_{1}\right) \cdots A_{\nu_{m}}\left(y_{m}\right)\right]_{l}^{k} .}
\end{array}
$$

The $m=0$ case should be interpreted as follows: it has vertices $W_{\mu_{1} \cdots \mu_{n}},\left(x_{1}, \cdots, x_{n} ; ; x, y\right)$ and the second product of gauge fields is replaced by $\delta^{k}{ }_{l}$. The $n=0$ case is defined similarly. We will write the $m=0$ vertices more compactly as

$$
W_{\mu_{1} \cdots \mu_{n}}\left(x_{1}, \cdots, x_{n} ; x, y\right) \equiv W_{\mu_{1} \cdots \mu_{n}},\left(x_{1}, \cdots, x_{n} ; ; x, y\right)
$$

In addition, the $m=n=0$ vertex is just the original kernel $(2.1 b)$, i.e.

$$
W,(; ; x, y) \equiv W_{x y}
$$




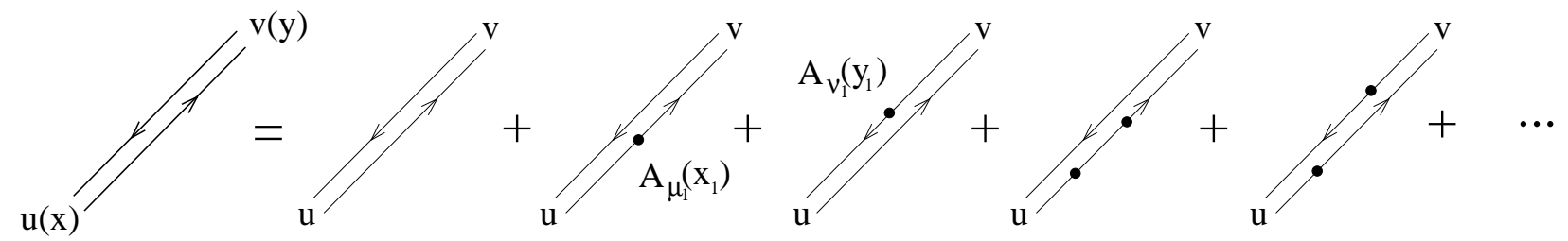

Fig.2. Expansion of the wine in gauge fields. The thick black lines stand for the full series.

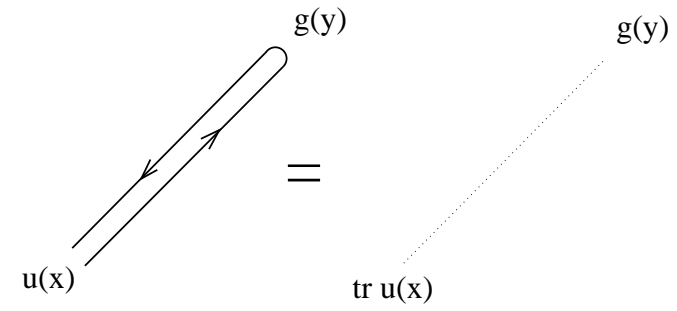

Fig.3. Coincident line identity. The dotted line is just the $W$ kernel $(2.1 b)$.

This expansion is illustrated in fig. 2.

Up to the requirement that it is still smooth in momentum space (i.e. that all vertices are Taylor expandable to all orders in momenta) [3] and some symmetry constraints ( $c f$. below (4.6) and later), the covariantization $\{W\}$ is of our choosing. However, for simplicity we further impose some 'coincident line' identities (described in sec.5) which in particular result in

$$
v_{k}^{j}(y)=\delta_{k}^{j} g(y) \quad \forall y \quad \Longrightarrow \quad u\{W\} v=(\operatorname{tr} u) \cdot W \cdot g \quad,
$$

as represented in fig. 3 .

For example, we could simply include in (2.1a) two straight Wilson lines

$$
u\{W\} v=\iint d^{D} x d^{D} y W_{x y} \operatorname{tr} u(x) \Phi\left[\mathcal{C}_{x y}\right] v(y) \Phi^{-1}\left[\mathcal{C}_{x y}\right],
$$

$\mathcal{C}_{x y}$ being the straight line between $x$ and $y$. (Later we will consider more general curves. Recall that the Wilson line is the path ordered exponential:

$$
\begin{aligned}
\Phi\left[\mathcal{C}_{x y}\right] & =P \exp -i \int_{\mathcal{C}_{x y}} d z^{\mu} A_{\mu}(z) \\
& =1-i \int_{0}^{1} d \tau \dot{z}^{\mu} A_{\mu}(z)-\int_{0}^{1} d \tau_{2} \int_{0}^{\tau_{2}} d \tau_{1} \dot{z} \cdot A\left(\tau_{1}\right) \dot{z} \cdot A\left(\tau_{2}\right)+\cdots
\end{aligned}
$$

where we have parametrized $\mathcal{C}_{x y}$ by $z^{\mu}(\tau), \tau \in[0,1], z(0)=x, z(1)=y$.) Another choice can be obtained by utilising the momentum representation, to write

$$
u\{W\} v=\operatorname{tr} \int d^{D} x u(x) W\left(-D^{2} / \Lambda^{2}\right) \cdot v(x)
$$


(where, as above (4.1), the covariant derivatives act by commutation). We will not need to specify the choice in this paper.

We covariantize (3.1) to

$$
\hat{S}=\frac{1}{2} F_{\mu \nu}\left\{c^{-1}\right\} F_{\mu \nu}
$$

(Note that gauge invariance forces us to include not just the Gaussian fixed point but also the interactions.) Our (insufficiently regularised) exact RG is then just the covariantization of (3.5) [3]:

$$
\Lambda \frac{\partial}{\partial \Lambda} S=-\frac{1}{2 \Lambda^{2}} \frac{\delta S}{\delta A_{\mu}}\left\{c^{\prime}\right\} \frac{\delta \Sigma_{g}}{\delta A_{\mu}}+\frac{1}{2 \Lambda^{2}} \frac{\delta}{\delta A_{\mu}}\left\{c^{\prime}\right\} \frac{\delta \Sigma_{g}}{\delta A_{\mu}} .
$$

Just as in (2.4), the first term on the RHS is the classical term, yielding the tree corrections, while the second, quantum, term, generates the loop corrections. Note that we have assumed the same functional relationship for the covariantization of $c^{-1}$ and $c^{\prime}[e . g$. as in (4.13)]. This is convenient but not necessary. The flow preserves the corresponding partition function (3.4), just as before, because (3.7) holds - on replacing $\cdot c^{\prime} \cdot$ with $\left\{c^{\prime}\right\}$.

The coupling, $g$, which appears in (4.15) through (3.6), is defined by $S$ via its unique derivative-squared term:

$$
S=\frac{1}{2 g^{2}} \operatorname{tr} \int d^{D} x F_{\mu \nu}^{2}+O\left(\partial^{3}\right)
$$

(discarding the vacuum energy). Note that $S$ must have a derivative expansion to all orders, because $\Lambda$ must play the rôle of an infrared cutoff in its vertices [23] [4], as explained below.

One of the great attractions of gauge theory is that in four dimensions, there are no relevant or marginal operators 5 we can put in by hand. In this case, $g$ is the only coupling, and furthermore the self-similarity of the continuum solution [4] ensures that the only explicit dimensionful parameter is $\Lambda$ whose appearance is then determined by dimensions. Thus in $D=4$ dimensions we can write for the continuum solution

$$
S=\frac{1}{2 g^{2}} \operatorname{tr} \int d^{4} x F_{\mu \nu}^{2}+O\left(\partial^{3} / \Lambda\right)
$$

(again discarding the vacuum energy).

In preparation for the large $N$ limit we write $g \mapsto g / \sqrt{N}, S \mapsto N S$. This has no effect on (3.6), 4.16) or 4.17), while 4.15) becomes

$$
\Lambda \frac{\partial}{\partial \Lambda} S=-\frac{1}{2 \Lambda^{2}} \frac{\delta S}{\delta A_{\mu}}\left\{c^{\prime}\right\} \frac{\delta \Sigma_{g}}{\delta A_{\mu}}+\frac{1}{N} \frac{1}{2 \Lambda^{2}} \frac{\delta}{\delta A_{\mu}}\left\{c^{\prime}\right\} \frac{\delta \Sigma_{g}}{\delta A_{\mu}}
$$

5 with respect to the Gaussian fixed point 
As we will show explicitly in the next section, the quantum term survives the large $N$ limit only in those terms in which each loop correction is accompanied by $\operatorname{tr} 1=N$ [3].

We finish this section by discussing some important qualitative criteria satisfied by (4.15) or (4.18). Recall that it is a fundamental requirement of the renormalization group that the Kadanoff blocking transformation [ [ 1 ] should affect variables only in a localised patch; long range interactions should appear only after infinitely many steps (analogously here $\Lambda \rightarrow 0)$.

On the one hand, obviously, we must therefore require that each RG step is free from infrared singularities, 6 equivalently here that the flow equation must have an all-orders Taylor expansion in small external momenta. We call this the requirement of 'quasilocality' [3]. This implies that the same is true of $S$, as we claimed above, providing only that we ensure that $\Lambda$-integration constants are also chosen quasilocal. As we will confirm explicitly, quasilocality then follows from the smoothness of the wine-vertices and $\hat{S}$. After integrating down to some intermediate scale $\Lambda$, the long range interactions are hidden in $S$ in this quasilocality (since the modes that are integrated out are infrared cutoff by $\Lambda$ ).

On the other hand, we want to ensure that unintended fundamental physics or even non-local non-physics is not also hidden in this quasilocality. We call this the requirement of 'ultralocality'. For momenta much larger than the infrared cutoff $\Lambda$, the typical $\sim 1 / p^{2}$ behaviour of fundamental propagating modes does show up in the vertices of $S$. Therefore the requirement of ultralocality corresponds to ensuring that there are no spurious inverse powers of momenta in this asymptotic expansion [3], in particular that we do not introduce any propagator-like terms into (4.14).

(Actually, despite the intuition, it is not clear to us whether this requirement is really needed. A more careful study than that reported in ref. [3], shows that it is not in fact possible to generate an arbitrary quasilocal $S^{0}$ from a quasilocal $\hat{S}$, rather there are some restrictions on additions to $S^{0}$. It may be that the physics is still correct but hidden in a change of variables. To definitively answer these issues requires generalising the recipe for extracting correlators and thus scattering matrix elements from the effective action [23] [4].)

Finally, we can see indirectly that (4.15) must still correspond to integrating out, once this flow equation is completely regularised, as follows. In this case, by definition all

6 Actually this statement is strictly true only for a smooth (i.e. infinitely differentiable) cutoff. More subtle cases, e.g. sharp cutoff [27], will not be considered here. 
momentum integrals are bounded. But since $\Lambda$ appears as the ultraviolet cutoff scale in these integrals, this means that contributions from momenta larger than some scale $q$ must vanish in the limit $q / \Lambda \rightarrow \infty$. Thus we see that as $\Lambda \rightarrow 0$, the remaining contribution from any fixed range of non-vanishing momentum scales, disappears. Since the partition function (3.4) is unchanged under the flow, the contributions from a given fixed momentum scale must still be in there somewhere, and the only other place they can be, is to already be encoded in the effective action - i.e. the modes have been integrated out. (Strictly it is the partition function (3.4) that we must show is completely regularised, and not just the flow equation. It is hard to see here however, how this property could separately fail.) A more intuitive, but incomplete, requirement for integrating out to be taking place (which is also satisfied) was discussed in ref. [3].

\section{Gauge field expansion and the large $\mathrm{N}$ limit}

The Wilsonian effective action $S$, being gauge invariant, has an expansion in traces and products of traces:

$$
\begin{aligned}
S & =\sum_{n=2}^{\infty} \frac{1}{n} \int d^{D} x_{1} \cdots d^{D} x_{n} S_{\mu_{1} \cdots \mu_{n}}\left(x_{1}, \cdots, x_{n}\right) \operatorname{tr} A_{\mu_{1}}\left(x_{1}\right) \cdots A_{\mu_{n}}\left(x_{n}\right) \\
& +\frac{1}{2 !} \sum_{m, n=2}^{\infty} \frac{1}{n m} \int d^{D} x_{1} \cdots d^{D} x_{n} d^{D} y_{1} \cdots d^{D} y_{m} S_{\mu_{1} \cdots \mu_{n}, \nu_{1} \cdots \nu_{m}}\left(x_{1}, \cdots, x_{n} ; y_{1}, \cdots, y_{m}\right) \\
& +\operatorname{tr} A_{\mu_{1}}\left(x_{1}\right) \cdots A_{\mu_{n}}\left(x_{n}\right) \operatorname{tr} A_{\nu_{1}}\left(y_{1}\right) \cdots A_{\nu_{m}}\left(y_{m}\right) \\
& +\cdots
\end{aligned}
$$

This is represented graphically in fig. 4 ( $c f$. fig. 2. Note that the combinatorics in the figures are those of Feynman rules: i.e. each diagram stands for the sum over all ways of assigning the gauge fields to the points, whilst respecting the order in the traces.)

The momentum space vertices are written as

$$
S_{\mu_{1} \cdots \mu_{n}}\left(p_{1}, \cdots, p_{n}\right)(2 \pi)^{D} \delta\left(\sum_{i=1}^{n} p_{i}\right)=\int d^{D} x_{1} \cdots d^{D} x_{n} \mathrm{e}^{-i \sum_{i} x_{i} \cdot p_{i}} S_{\mu_{1} \cdots \mu_{n}}\left(x_{1}, \cdots, x_{n}\right)
$$

where all momenta are taken pointing into the vertex, and similarly for the vertices in

(4.7). $\hat{S}$ has an expansion in only single trace vertices $\hat{S}_{\mu_{1} \cdots \mu_{n}}\left(x_{1}, \cdots, x_{n}\right)$, as follows from 


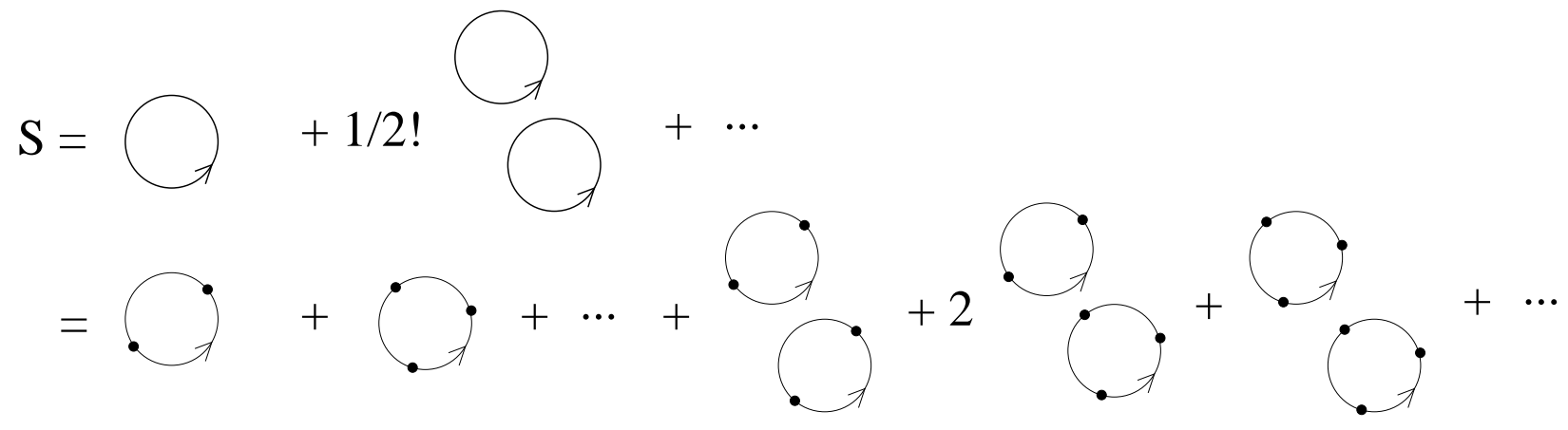

Fig.4. Expansion of the action in traces, and products of traces, of gauge fields.

(4.14). In fact, combining (4.7) and (4.14), we may easily read off expressions for these vertices [3]:

$$
\begin{aligned}
\hat{S}_{\mu \nu}(p) \equiv & \hat{S}_{\mu \nu}(p,-p)=2 \Delta_{\mu \nu}(p) / c_{p} \\
\hat{S}_{\mu \nu \lambda}(p, q, r)= & \frac{2}{c_{p}}\left(p_{\lambda} \delta_{\mu \nu}-p_{\nu} \delta_{\lambda \mu}\right)+2 c_{\nu}^{-1}(q ; p, r)\left(p_{\lambda} r_{\mu}-p . r \delta_{\lambda \mu}\right)+\text { cycles } \\
\hat{S}_{\mu \nu \lambda \sigma}(p, q, r, s)= & \frac{1}{c_{p+q}}\left(\delta_{\sigma \mu} \delta_{\lambda \nu}-\delta_{\lambda \mu} \delta_{\nu \sigma}\right)+2 c_{\nu}^{-1}(q ; p, r+s)\left(p_{\sigma} \delta_{\lambda \mu}-p_{\lambda} \delta_{\sigma \mu}\right) \\
& +2 c_{\sigma}^{-1}(s ; p, r+q)\left(p_{\nu} \delta_{\mu \lambda}-p_{\lambda} \delta_{\mu \nu}\right)+2 c_{\nu \lambda}^{-1}(q, r ; p, s)\left(p_{\sigma} s_{\mu}-p . s \delta_{\sigma \mu}\right) \\
& +c_{\nu, \sigma}^{-1}(q ; s ; p, r)\left(p_{\lambda} r_{\mu}-p \cdot r \delta_{\lambda \mu}\right)+\text { cycles }
\end{aligned}
$$

etc. , where in the two-point vertex we set $p_{1}=-p_{2}=p$, and introduce the shorthand $c_{p} \equiv c\left(p^{2} / \Lambda^{2}\right)$ and the transverse combination $\Delta_{\mu \nu}(p):=p^{2} \delta_{\mu \nu}-p_{\mu} p_{\nu}$, in the three-point vertex we add the two cyclic permutations of $\left(p_{\mu}, q_{\nu}, r_{\lambda}\right)$, and in the four-point vertex the three cyclic permutations of $\left(p_{\mu}, q_{\nu}, r_{\lambda}, s_{\sigma}\right)$. Note that, since $c_{p}$ and all the wine-vertices are smooth, all the $\hat{S}$-vertices have the same property.

Concentrating on the single trace terms of $S$ (or $\hat{S}$ ) we see from (4.7), (5.1), and (4.4), that $\left\{c^{\prime}\right\} \delta S / \delta A_{\mu}$ opens up the trace by removing one $A_{\mu}$ and replacing it with the ends of the lines of parallel transport in fig. 1. In the classical term of (4.18) the leftmost $\delta / \delta A_{\mu}$ does the same with another (copy of the) action, while in the quantum term, the leftmost $\delta / \delta A_{\mu}$ opens up the same trace again and attaches the other ends of the parallel transport lines, splitting the trace in two, as in (4.5). Diagrammatically, the single trace terms of $S$, under a small change in $\Lambda$, thus induce the contributions in fig. 5 .

Strictly speaking we should include in the quantum term of (4.18), the contributions as in fig. 6 where the leftmost functional derivative attacks the parallel transport lines 

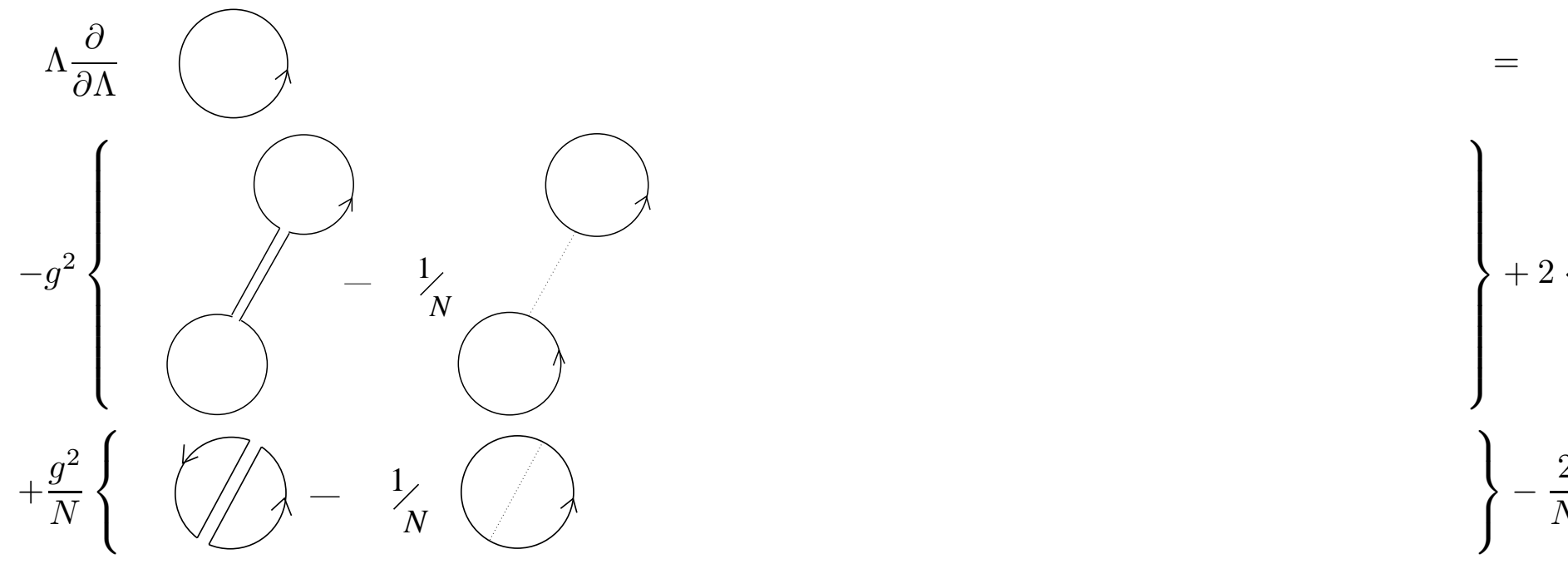

Fig.5. Diagrammatic representation of the flow equation. A circumflex in the circle stands for $\hat{S}$, a dotted line for attachment of $c_{x y}^{\prime}$ only, as in fig. 3 .

themselves. However, we can avoid them by a limiting procedure keeping the position of the functional derivative away from the wine [3]. In fact these terms will vanish once the Pauli-Villars contributions are included [9].

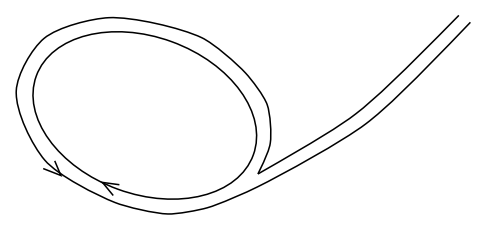

Fig.6. A wine biting its own tail.

As $\Lambda \rightarrow \infty, S$ is given by just the first term in (4.17), and thus is a single trace in this limit. We see from fig. 5 that those terms with a product of two traces are down by factors of $1 / N$. In the large $N$ limit the only quantum terms that survive are the split-open traces where all the gauge fields lie in only one of the two new traces, as in fig. 7 . Thus in the large $N$ limit, the single trace property is preserved by the flow and the effective action at any $\Lambda$ contains only single trace terms.

However, whilst it is true that the large $N$ limit of the effective action is a single trace, the limit of the flow equation itself may be more subtle: two-trace terms with just two gauge fields in one of the traces are also formally down by a factor of $1 / N$, but the attachment of a field-free wine to these two gauge fields in a higher order quantum correction, will 


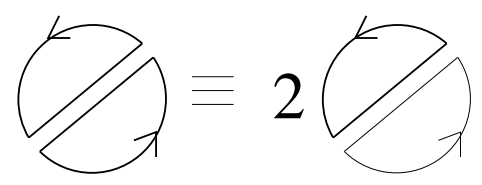

Fig.7. In the large $N$ limit, the surviving terms have all gauge fields living on one of the two traces, the no-gauge-fields trace contributing $\operatorname{tr} 1=N$.

then yield a further factor of $1 / N$ (for the quantum correction) and a factor of $N^{2}$ for two field-free traces, thus contributing in principle a finite amount in the $N \rightarrow \infty$ limit. To one loop this does not happen because two-trace terms with just two fields in one of the traces, vanish at tree level for symmetry reasons, as we prove in sec. 8 . We leave to the future, the question of whether or not this more subtle large $N$ limit of the flow equations takes place at higher loop order.

\section{Wilson loop representation}

The Wilson loop representation was central to our exposition in [3], and indeed plays a powerful rôle in our own thinking, but we have intentionally avoided introducing it until this point to emphasise that the flow equations and their properties hold entirely separately from this representation, even though we find that these properties show up most clearly through this representation.

We have already mentioned, $c f$. (4.11), that we could covariantize the kernel by using straight Wilson lines. More generally, we could use curves $\mathcal{C}_{x y}$, although to ensure Lorentz invariance these would have to be averaged over their orientations. The most general covariantization, preserving the fact that in (4.7) the gauge fields appear in just two strings, is then given by (path) integrals and/or sums over all the configurations of these two curves, with a normalised measure of our own choosing [up to the Lorentz covariance requirement above, the smoothness requirement above (4.10), and exchange symmetry below (4.6)]. However, we simplify this by traversing back along a coincident Wilson line, i.e. back from $y$ to $x$ along the same curve:

$$
u\{W\} v=\iint d^{D} x d^{D} y \int \mathcal{D} \mathcal{C}_{x y} \operatorname{tr} u(x) \Phi\left[\mathcal{C}_{x y}\right] v(y) \Phi^{-1}\left[\mathcal{C}_{x y}\right]
$$

The measure (hidden in the definition of $\mathcal{D} \mathcal{C}_{x y}$ ) does not of course depend on the parametrization of the path, and is normalised by

$$
\int \mathcal{D} \mathcal{C}_{x y} 1=W_{x y}
$$


as follows from (4.9). (As a trivial example, in the case of (4.11), it has support only on the straight line $\mathcal{C}_{x y}$ where the measure collapses to multiplication by $W_{x y}$.) Clearly (6.1) and (6.2) imply the identity (4.10) we introduced earlier.

Similarly the most general form for the action is in terms of path integrals over Wilson loops $\varphi[\mathcal{C}]=\operatorname{tr} \Phi\left[\mathcal{C}_{x x}\right]\left(\mathcal{C}\right.$ being a closed contour, $x$ some arbitrary point on it, and $\mathcal{C}_{x x}$ being the marked contour starting and finishing at $x$ ). The most general single trace term is obtained from an integral over all configurations of a single Wilson loop, the most general two-trace terms from two Wilson loops and so on. Thus (5.1) may be represented as

$$
S=\int \mathcal{D C} \varphi[\mathcal{C}]+\frac{1}{2 !} \int \mathcal{D}\left[\mathcal{C}_{1}, \mathcal{C}_{2}\right] \varphi\left[\mathcal{C}_{1}\right] \varphi\left[\mathcal{C}_{2}\right]+\cdots
$$

and $\hat{S}$ may be written

$$
\hat{S}=\int \mathcal{D} \hat{\mathcal{C}} \varphi[\hat{\mathcal{C}}]
$$

[by (3.1)]. While the measure $\mathcal{D} \hat{\mathcal{C}}$ is of our choosing, the measures $\mathcal{D C}, \mathcal{D}\left[\mathcal{C}_{1}, \mathcal{C}_{2}\right]$, etc. , are determined by the flow equations.

By virtue of the fact that

$$
\delta \Phi\left[\mathcal{C}_{x y}\right]=-i \int_{\mathcal{C}_{x y}} d z^{\mu} \Phi\left[\mathcal{C}_{x z}\right] \delta A_{\mu}(z) \Phi\left[\mathcal{C}_{z y}\right]
$$

where $\mathcal{C}_{x z}\left(\mathcal{C}_{z y}\right)$ is the part of $\mathcal{C}_{x y}$ before (after) $z$, all our previous figures still hold but as 'snapshots' of the Wilson loops and/or lines. We only have to remember that wherever the 'wine' attaches, it is integrated around the curve by $-i \int d z^{\mu}$. As a result, our previous figures, e.g. fig. 5, are no longer just 'hieroglyphics' -indicating the form of the resulting traces - but come 'alive' as fluctuating Wilson loops (or lines) i.e. representatives of the appropriate measures $($ e.g. $\mathcal{D C})$.

From the previous section, in the large $N$ limit, (6.3) collapses from a 'gas' of Wilson loops to just a single path integral over one Wilson loop. We can write this explicitly as a path integral over a single particle circulating in a loop:

$$
S=P \int \mathcal{D} x \mathrm{e}^{-s[x]+\oint d x \cdot A}
$$

where we have parametrized the closed curve $\mathcal{C}$ by $x^{\mu}(\tau)$, and the (parametrization independent) path integral measure $\mathcal{D} x \mathrm{e}^{-s}$ is determined by the flow equation. Note that fig. 5 indicates that this measure is rather unusual compared to that e.g. of a free particle, 
but nevertheless we see that gauge theory in the large $N$ limit, is equivalent to a form of quantum mechanics for a single particle!

Combining (6.3) and (5.2), yields Wilson loop expressions for the vertices, e.g. the single trace vertices are given as [3]

$$
S_{\mu_{1} \cdots \mu_{n}}\left(p_{1}, \cdots, p_{n}\right)(2 \pi)^{D} \delta\left(\sum_{i=1}^{n} p_{i}\right)=(-i)^{n} \int \mathcal{D C} \oint_{(1,2, \cdots, n)} \cdots \oint d x_{1}^{\mu_{1}} d x_{2}^{\mu_{2}} \cdots d x_{n}^{\mu_{n}} \mathrm{e}^{-i \sum_{i} x_{i} \cdot p_{i}}
$$

(similarly $\hat{S}$ and $\hat{\mathcal{C}}$ ) where the notation $(1,2, \cdots, n)$ stands for integrating round the loop while preserving the cyclical order, and similarly (6.1) and (4.7) give

$$
\begin{array}{r}
W_{\mu_{1} \cdots \mu_{n}, \nu_{1} \cdots \nu_{m}}\left(p_{1}, \cdots, p_{n} ; q_{1}, \cdots, q_{m} ; r, s\right)(2 \pi)^{D} \delta\left(\sum_{i=1}^{n} p_{i}+\sum_{j=1}^{m} q_{j}+r+s\right)= \\
(-i)^{n+m} \iint d^{D} u d^{D} v \int \mathcal{D} \mathcal{C}_{u v} \int_{u}^{v} d x_{n}^{\mu_{n}} \int_{u}^{x_{n}} d x_{n-1}^{\mu_{n}-1} \cdots \int_{u}^{x_{2}} d x_{1}^{\mu_{1}} \int_{v}^{u} d y_{m}^{\nu_{m}} \int_{v}^{y_{m}} d y_{m-1}^{\nu_{m}-1} \cdots \int_{v}^{y_{2}} d y_{1}^{\nu_{1}} \\
\exp -i\left(r . u+s . v+\sum_{i} p_{i} . x_{i}+\sum_{j} q_{j} \cdot y_{j}\right)
\end{array}
$$

where the $x_{i}$ integration is along the curve $\mathcal{C}_{u v}$, and the $y_{j}$ integration along the same curve but in the opposite direction $c f$. fig. 2 or fig. 8 .

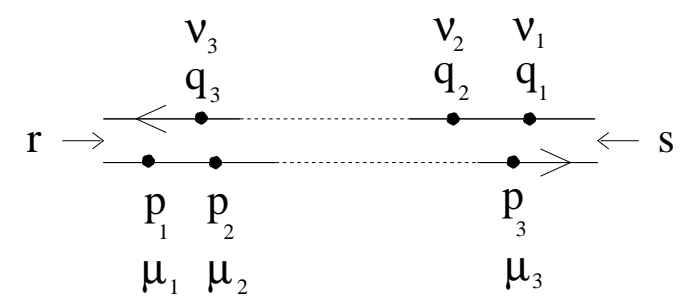

Fig.8. Feynman rule for the wine, with momentum labels.

As we will show now in some small examples, these Wilson loop (line) representations allow one easily to extract many general properties of the solutions.

\section{Symmetries}

The gauge invariance (trivial Ward) identities follow by applying $\delta A_{\mu}=\partial_{\mu} \omega-i\left[A_{\mu}, \omega\right]$ to (5.1) and (4.7), or more simply by direct integration in (6.5) and (6.6):

$$
p_{1}^{\mu_{1}} S_{\mu_{1} \cdots \mu_{n}}\left(p_{1}, \cdots, p_{n}\right)=S_{\mu_{2} \cdots \mu_{n}}\left(p_{1}+p_{2}, p_{3}, \cdots, p_{n}\right)-S_{\mu_{2} \cdots \mu_{n}}\left(p_{2}, \cdots, p_{n-1}, p_{n}+p_{1}\right)
$$


Note that it is clear from (5.1) or (6.5) that these vertices are cyclically symmetric:

$$
S_{\mu_{1} \cdots \mu_{n}}\left(p_{1}, \cdots, p_{n}\right)=S_{\mu_{2} \cdots \mu_{n} \mu_{1}}\left(p_{2}, \cdots, p_{n}, p_{1}\right)
$$

Diagrammatically, (7.1) corresponds to a 'push forward' minus a 'pull back' of the point concerned to the end points of the relevant integration domain in (6.5), as in fig. 9.

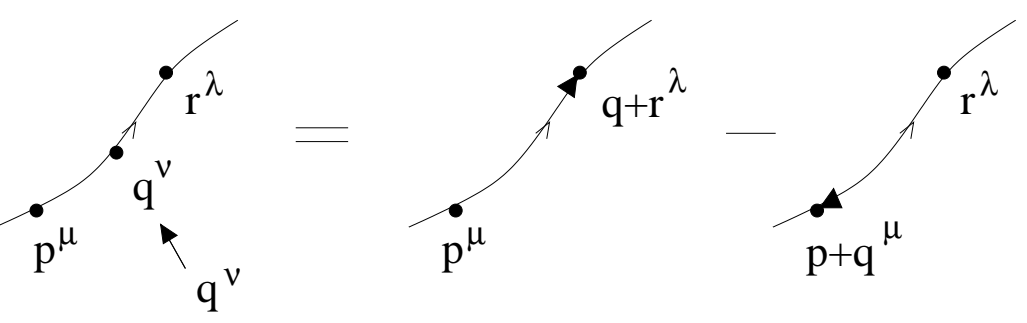

Fig.9. Graphical representation of gauge invariance identities.

Of course, these same properties hold for $\hat{S}_{\mu_{1} \cdots \mu_{n}}\left(p_{1}, \cdots, p_{n}\right)$, and separately for each string in $S_{\mu_{1} \cdots \mu_{n}, \nu_{1} \cdots \nu_{m}}\left(p_{1}, \cdots, p_{n} ; q_{1}, \cdots, q_{m}\right)$, the two-trace vertices of (5.1). (In addition

$$
S_{\mu_{1} \cdots \mu_{n}, \nu_{1} \cdots \nu_{m}}\left(p_{1}, \cdots, p_{n} ; q_{1}, \cdots, q_{m}\right)=S_{\nu_{1} \cdots \nu_{m}, \mu_{1} \cdots \mu_{n}}\left(q_{1}, \cdots, q_{m} ; p_{1}, \cdots, p_{n}\right)
$$

without loss of generality, from (5.1) or (6.3).) For the wine vertices we have the analogous identites except that outer momenta can reach the end of line:

$$
\begin{aligned}
p_{1}^{\mu_{1}} W_{\mu_{1} \cdots \mu_{n}, \nu_{1} \cdots \nu_{m}}\left(p_{1}, \cdots, p_{n} ; q_{1}, \cdots, q_{m} ; r, s\right) & \\
& W_{\mu_{2} \cdots \mu_{n}, \nu_{1} \cdots \nu_{m}}\left(p_{1}+p_{2}, p_{3}, \cdots, p_{n} ; q_{1}, \cdots, q_{m} ; r, s\right) \\
& -W_{\mu_{2} \cdots \mu_{n}, \nu_{1} \cdots \nu_{m}}\left(p_{2}, \cdots, p_{n} ; q_{1}, \cdots, q_{m} ; r+p_{1}, s\right)
\end{aligned}
$$

with similar identities for contraction with $p_{n}^{\mu_{n}}, q_{1}^{\nu_{1}}$ and $q_{m}^{\nu_{m}}$, as is clear from fig. 8 .

Charge conjugation (C) invariance follows from the symmetry $A_{\mu} \leftrightarrow-A_{\mu}^{T}$, equivalently from reversal of the direction of all Wilson loops (lines), thus

$$
S_{\mu_{1} \cdots \mu_{n}}\left(p_{1}, \cdots, p_{n}\right)=(-)^{n} S_{\mu_{n} \cdots \mu_{1}}\left(p_{n}, \cdots, p_{1}\right) \quad,
$$

(similarly $\hat{S}$ ) and combining C invariance with the exchange identity,

$$
W_{\mu_{1} \cdots \mu_{n}, \nu_{1} \cdots \nu_{m}}\left(p_{1}, \cdots, p_{n} ; q_{1}, \cdots, q_{m} ; r, s\right)=W_{\nu_{1} \cdots \nu_{m}, \mu_{1} \cdots \mu_{n}}\left(q_{1}, \cdots, q_{m} ; p_{1}, \cdots, p_{n} ; s, r\right)
$$


(which one easily sees by rotating fig. 8 or from (4.6) under $u \leftrightarrow v$ ), gives

$$
\begin{aligned}
& W_{\mu_{1} \cdots \mu_{n}, \nu_{1} \cdots \nu_{m}}\left(p_{1}, \cdots, p_{n} ; q_{1}, \cdots, q_{m} ; r, s\right)= \\
& (-)^{n+m} W_{\mu_{n} \cdots \mu_{1}, \nu_{m} \cdots \nu_{1}}\left(p_{n}, \cdots, p_{1} ; q_{m}, \cdots, q_{1} ; s, r\right)
\end{aligned}
$$

Lorentz invariance implies that changing the sign of all the momentum arguments in the vertices (of $S, \hat{S}$ or $W$ ) just changes the sign of those with an odd number of momentum arguments and leaves alone those with an even number.

Finally the 'coincident line' identities readily follow from (6.6) by changing the direction of the $y$ integration:

$$
W_{\mu_{1} \cdots \mu_{n}, \nu_{1} \cdots \nu_{m}}\left(p_{1}, \cdots, p_{n} ; q_{1}, \cdots, q_{m} ; r, s\right)=(-)^{m} \sum_{\text {interleaves }} W_{\lambda_{1} \cdots \lambda_{m+n}}\left(k_{1}, \cdots, k_{m+n} ; r, s\right)
$$

where we have used (4.8), and the sum runs over all interleaves of the sequences $p_{1}^{\mu_{1}}, \cdots, p_{n}^{\mu_{n}}$ and $q_{m}^{\nu_{m}}, \cdots, q_{1}^{\nu_{1}}$ i.e. combined sequences $k_{1}^{\lambda_{1}}, \cdots, k_{m+n}^{\lambda_{m+n}}$ in which the $p^{\mu_{\mathrm{S}}}$ remain ordered with respect to each other, and similarly the $q^{\nu}$ s remain in reverse order.

Some useful examples are:

$S_{\mu \nu \lambda}(p, q, r)$ is antisymmetric under exchange of any pair in $\left(p^{\mu}, q^{\nu}, r^{\lambda}\right)$,

$$
\begin{aligned}
W_{\mu}(p ; q, r) & =-W_{\mu}(p ; r, q) \quad, \quad p^{\mu} W_{\mu}(p ; q, r)=W_{q}-W_{r} \\
S_{\mu \nu \lambda \sigma}(p, q, r, s) & =S_{\nu \mu \sigma \lambda}(q, p, s, r) \quad, \quad W_{\mu \nu}(p, q ; r, s)=W_{\nu \mu}(q, p ; s, r) \\
W_{; \nu_{1} \cdots \nu_{m}}\left(; q_{1}, \cdots, q_{m} ; r, s\right) & =W_{\nu_{1} \cdots \nu_{m}}\left(q_{1}, \cdots, q_{m} ; s, r\right) \\
W_{\mu, \nu}(p ; q ; r, s) & =-W_{\mu \nu}(p, q ; r, s)-W_{\nu \mu}(q, p ; r, s)
\end{aligned}
$$

\section{Perturbative expansion}

Although we will be especially interested in four dimensions, we keep $D \neq 4$ here since it will be helpful to access this via the limit $D \rightarrow 4$ [3]. It will also be helpful to write (4.18) as

$$
\Lambda \frac{\partial}{\partial \Lambda} S=-a_{0}\left[S, g^{2} S-2 \hat{S}\right]+a_{1}\left[g^{2} S-2 \hat{S}\right]
$$

where we have used (3.6) and written the classical term as the bilinear functional $-a_{0}$ and the quantum term as the linear functional $a_{1} .^{\dagger}$ At the classical level, we see from

$\dagger$ The subscripts indicate the order of $\hbar$, i.e. the number of loops involved. 
(4.16) and (8.1), that $S \sim 1 / g^{2}$. Substituting this in the quantum term of (8.1), we see that the one loop correction $\sim g^{0}$, and so on. Thus by iteration, $S$ has the weak coupling $\operatorname{expansion}^{\dagger}$

$$
S=\frac{1}{g^{2}} S_{0}+S_{1}+g^{2} S_{2}+\cdots
$$

This is of course the form expected by the usual graphical considerations [after performing $A_{\mu} \mapsto A_{\mu} / g$ as above (3.3)]. Substituting this expansion in (8.1) and recalling that $g$ will run at the quantum level, we see that the $\beta$ function must also take the standard form ${ }^{\dagger}$

$$
\beta:=\Lambda \frac{\partial g}{\partial \Lambda}=\beta_{1} g^{3}+\beta_{2} g^{5}+\cdots
$$

We will see below how the $\beta_{i}$ are determined. From (8.2) and (8.3), we obtain the loopwise expansion of (8.1):

$$
\begin{aligned}
& \Lambda \frac{\partial}{\partial \Lambda} S_{0}=-a_{0}\left[S_{0}, S_{0}-2 \hat{S}\right] \\
& \Lambda \frac{\partial}{\partial \Lambda} S_{1}=2 \beta_{1} S_{0}-2 a_{0}\left[S_{0}-\hat{S}, S_{1}\right]+a_{1}\left[S_{0}-2 \hat{S}\right] \\
& \Lambda \frac{\partial}{\partial \Lambda} S_{2}=2 \beta_{2} S_{0}-2 a_{0}\left[S_{0}-\hat{S}, S_{2}\right]-a_{0}\left[S_{1}, S_{1}\right]+a_{1}\left[S_{1}\right]
\end{aligned}
$$

etc. We mention in passing that by considering linear perturbations $S \mapsto S+\epsilon \mathcal{O}$, we easily obtain the flow equations for the integrated operator $\mathcal{O}$, and its weak coupling expansion. Thus from (8.1),

$$
\Lambda \frac{\partial}{\partial \Lambda} \mathcal{O}=-2 a_{0}\left[g^{2} S-\hat{S}, \mathcal{O}\right]+g^{2} a_{1}[\mathcal{O}]
$$

From (8.2), we expand $\mathcal{O}=\frac{1}{g^{2}} \mathcal{O}_{0}+\mathcal{O}_{1}+g^{2} \mathcal{O}_{2}+\cdots$ giving, either from (8.3), (8.2) and (8.1), or directly from 8.4$)-(8.6)$ :

$$
\begin{aligned}
& \Lambda \frac{\partial}{\partial \Lambda} \mathcal{O}_{0}=-2 a_{0}\left[S_{0}-\hat{S}, \mathcal{O}_{0}\right] \\
& \Lambda \frac{\partial}{\partial \Lambda} \mathcal{O}_{1}=2 \beta_{1} \mathcal{O}_{0}-2 a_{0}\left[S_{0}-\hat{S}, \mathcal{O}_{1}\right]-2 a_{0}\left[S_{1}, \mathcal{O}_{0}\right]+a_{1}\left[\mathcal{O}_{0}\right] \\
& \Lambda \frac{\partial}{\partial \Lambda} \mathcal{O}_{2}=2 \beta_{2} \mathcal{O}_{0}-2 a_{0}\left[S_{0}-\hat{S}, \mathcal{O}_{2}\right]-2 a_{0}\left[S_{2}, \mathcal{O}_{0}\right]-2 a_{0}\left[S_{1}, \mathcal{O}_{1}\right]+a_{1}\left[\mathcal{O}_{1}\right]
\end{aligned}
$$

etc. 


\subsection{Feynman diagrammatics and the classical vertices}

Our diagrams, as in fig. 5, play one final rôle: expanded as in fig. 2, they yield Feynman diagrams which may be translated directly into the corresponding equations for the vertices. The translation rule is just that we must place the points and their associated momenta in all places on composite Wilson loops, whilst preserving the cyclic order. We then read off the appropriate wine and action vertices (applying momentum conservation -and including momentum integrals if appropriate), contract the Lorentz indices of points joined by wines and, from (4.18), multiply the whole by $1 / 2 \Lambda^{2}$. In the following we compute in this way the first few classical vertices $S_{\mu_{1} \cdots \mu_{n}}^{0}$.

The diagrams for the classical two-point vertex $S_{\mu \nu}^{0}(p)$ follow from (8.4) and the top two lines of fig. 5:

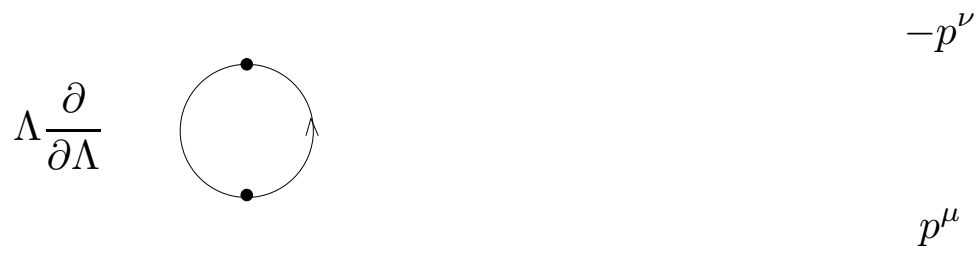

Fig.10. Feynman diagrams for the two-point vertex.

Here, the empty circle corresponds to $S_{0}$, not $S$ as in fig. 5 , and we have noted that since actions' one-point vertices vanish (by $\operatorname{tr} A_{\mu}=0$ but see also [3]), we must have at least one blob per lobe. Thus

$$
\Lambda \frac{\partial}{\partial \Lambda} S_{\mu \nu}^{0}(p)=-\frac{1}{2 \Lambda^{2}} c_{p}^{\prime}\left[S_{\mu \lambda}^{0}(p)-2 \hat{S}_{\mu \lambda}(p)\right] S_{\lambda \nu}^{0}(p)+\left(p_{\mu} \leftrightarrow-p_{\nu}\right)
$$

where similarly to $(5.3), c_{p}^{\prime} \equiv c^{\prime}\left(p^{2} / \Lambda^{2}\right)$. By gauge invariance and dimensions,

$$
S_{\mu \nu}^{0}(p)=2 \Delta_{\mu \nu}(p) / f\left(p^{2} / \Lambda^{2}\right)
$$

From (8.2), we require $f(0)=1$ so as to be consistent with (4.16) in the $g \rightarrow 0$ limit. Substituting (5.3), we readily find the unique solution to be $f=c$, and thus

$$
S_{\mu \nu}^{0}(p)=\hat{S}_{\mu \nu}(p)
$$

7 For convenience we move the $\hbar$ counting index to a superscript 
Similarly, from (8.4) and the top two lines of fig. 5, we obtain the following diagrams for the three-point vertex:

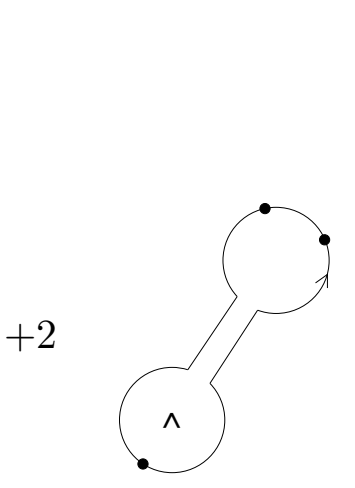

Fig.11. Feynman diagrams for the three-point vertex.

Using (8.13), the entire second line vanishes and we are left on the RHS only with terms that are already determined. We can thus immediately integrate to get

$$
\begin{aligned}
S_{\mu \nu \lambda}^{0}(p, q, r)= & -\int_{\Lambda}^{\infty} \frac{d \Lambda_{1}}{\Lambda_{1}^{3}}\left\{c_{r}^{\prime} \hat{S}_{\mu \nu \alpha}(p, q, r) \hat{S}_{\alpha \lambda}(r)+c_{\nu}^{\prime}(q ; p, r) \hat{S}_{\mu \alpha}(p) \hat{S}_{\alpha \lambda}(r)\right\} \\
& +2\left(r_{\nu} \delta_{\mu \lambda}-r_{\mu} \delta_{\nu \lambda}\right) \quad+\text { cycles } .
\end{aligned}
$$

Here it should be understood that in the curly brackets we replace $\Lambda$ with $\Lambda_{1}$, and to the whole expression we add the two cyclic permutations of $\left(p_{\mu}, q_{\nu}, r_{\lambda}\right)$. In principle the top limit would be $\Lambda_{0}$, the bare cutoff scale, but since the integrands have Taylor expansions in momenta $/ \Lambda_{1}$, the continuum limit $\Lambda_{0} \rightarrow \infty$ trivially exists (as expected at the classical level). The integration constant is a term independent of $\Lambda$, which by dimensions and locality ('quasi' or 'ultra', cf. sec. 4) must be linear in the momenta. By gauge invariance it has to be the unique covariantization of $2 \Delta_{\mu \nu}$ (see below), i.e. the three-point vertex in $\frac{1}{2} \int d^{D} x F_{\mu \nu}^{2}$. In fact, this conclusion follows directly by comparing the $\Lambda \rightarrow \infty$ limit of (4.17) with (8.14).

Using (7.1) and (7.2), one may readily check the gauge invariance of (8.14): contracting the RHS of (8.14) with $p^{\mu}$, we obtain

$$
-\int_{\Lambda}^{\infty} \frac{d \Lambda_{1}}{\Lambda_{1}^{3}}\left\{c_{r}^{\prime} \hat{S}_{\nu \alpha}(r) \hat{S}_{\alpha \lambda}(r)-c_{q}^{\prime} \hat{S}_{\lambda \alpha}(q) \hat{S}_{\alpha \nu}(q)\right\}+2 \Delta_{\nu \lambda}(r)-2 \Delta_{\nu \lambda}(q)
$$

after cancellation of some 'corner' terms containing $\hat{S}_{\nu \alpha}(q) \hat{S}_{\alpha \lambda}(r)$ and $c_{r}^{\prime}$ or $c_{q}^{\prime}$. From (8.11) and (8.13), the integrand is a total derivative integrating to boundary terms 
$\left[\hat{S}_{\nu \lambda}(r)-\hat{S}_{\nu \lambda}(q)\right]_{\Lambda}^{\infty}$. The top limit cancels the $\Delta$ terms in (8.15) if and only if the integration constant in (8.14) is a gauge covariantization of $2 \Delta_{\mu \nu}(p)$, while the bottom limit gives $S_{\nu \lambda}^{0}(r)-S_{\nu \lambda}^{0}(q)$ evaluated at $\Lambda$, as required. In fact, this check is clear essentially diagrammatically, using fig. 9, fig. 11, and fig. 10 as follows. On the RHS of fig. 11, we sum the results of pushing forward and pulling back each point in turn (i.e. summing over the cyclic orderings of the momentum $p^{\mu}$ ). The cancelling terms mentioned above, correspond to terms that meet at a corner where the wine joins $\hat{S}$ - push-forwards to the end of a wine cancelling pull-backs to this point in $\hat{S}$, and vice versa - while the remaining terms represent the integrand in (8.15). These appear as the two-point diagrams of fig. 10, with each point in turn replaced by the 'pushforward' and 'pullback' arrows of fig. 9 .

The simplification that the flow depends only on already known terms persists to all higher point classical vertices (and therefore also to all orders in $\hbar, a . k . a . g$ ): Since we need at least one blob per lobe, the flow of a classical $n$-point vertex cannot depend on a higherpoint vertex, and thus these equations are closed. Furthermore, when one lobe corresponds to an $n$-point $S^{0}$-vertex, the other corresponds to the two-point vertex (8.13). Since for $n>2$, there are two such terms in $a_{0}\left[S^{0}, S^{0}\right]$ but only one in $a_{0}\left[S^{0}, \hat{S}\right]$, these contributions cancel in (8.4). Thus the flow of $S_{\mu_{1} \cdots \mu_{n}}^{0}$ for $n>2$, depends only on lower-point vertices or $\hat{S}$, and may be immediately integrated.

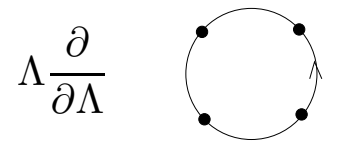

Fig.12. Feynman diagrams for the four-point vertex.

In a similar way, we compute the classical gauge invariant continuum four-point vertex. 
After simplifying by (8.13), the diagrams are those in fig. 12, thus

$$
\begin{aligned}
& S_{\mu \nu \lambda \sigma}^{0}(p, q, r, s)=-\int_{\Lambda}^{\infty} \frac{d \Lambda_{1}}{\Lambda_{1}^{3}}\left\{c_{p+q}^{\prime}\left(\hat{S}_{\mu \nu \alpha}(p, q, r+s)-\frac{1}{2} S_{\mu \nu \alpha}^{0}(p, q, r+s)\right) S_{\alpha \lambda \sigma}^{0}(p+q, r, s)\right. \\
& \quad+\hat{S}_{\sigma \alpha}(s) \hat{S}_{\mu \nu \alpha}(p, q, r+s) c_{\lambda}^{\prime}(r ; p+q, s)+\hat{S}_{\lambda \alpha}(r) \hat{S}_{\mu \nu \alpha}(p, q, r+s) c_{\sigma}^{\prime}(s ; r, p+q) \\
& \quad+\hat{S}_{\mu \alpha}(p) \hat{S}_{\alpha \sigma}(s) c_{\nu \lambda}^{\prime}(q, r ; p, s)+\frac{1}{2} \hat{S}_{\mu \alpha}(p) \hat{S}_{\alpha \lambda}(r) c_{\nu, \sigma}^{\prime}(q ; s ; p, r) \\
& \left.\quad+c_{s}^{\prime} \hat{S}_{\sigma \alpha}(s) \hat{S}_{\mu \nu \lambda \alpha}(p, q, r, s)+\operatorname{cycles}\right\}+2 \delta_{\mu \sigma} \delta_{\nu \lambda}-4 \delta_{\mu \lambda} \delta_{\nu \sigma}+2 \delta_{\mu \nu} \delta_{\lambda \sigma}
\end{aligned}
$$

where 'cycles' stands for the three cyclic permutations of $\left(p_{\mu}, q_{\nu}, r_{\lambda}, s_{\sigma}\right)$. We see that, as predicted in sect.5, (8.13), (8.14) and (8.16) are smooth in momenta.

Whilst (8.16) may look complicated, the expression is actually completely determined from the top term by gauge invariance and cyclicity: Indeed, the top term is sufficient to identify the two composite Wilson loops in (8.4), and from the graphical arguments below (8.15), gauge invariance then holds only if all the other terms appear with their correct coefficients, since these correspond to all cyclic order preserving positions of the points on the underlying composite Wilson loop, and only then do all the 'corner' cancellations correctly take place. Similarly all the other (e.g. higher-loop and/or higher-point) $S$ vertices follow from just one or two of their individual terms. In fact, since (4.14) is also a composite Wilson loop (6.4) [3], these same comments apply to the $\hat{S}$-vertices (5.3).

At finite $N$, classical vertices with more than one trace do exist. However, simply by $\operatorname{tr} A_{\mu}=0$, there can be no such vertices with three or less points. The four-point vertex $S_{\mu \nu, \lambda \sigma}^{0}(p, q ; r, s)$ actually also vanishes. Its flow is shown diagrammatically in fig. 13 , where once again we have simplified with $(8.13)$.

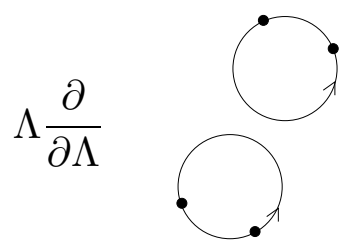

Fig.13. Feynman diagrams for the double-trace four-point vertex.

These contributions vanish on adding the cyclic permutations $p^{\mu} \leftrightarrow q^{\nu}$ or $r^{\lambda} \leftrightarrow s^{\sigma}$, by the antisymmetry of the three-point vertices [as in (7.4)]. Therefore $S_{\mu \nu, \lambda \sigma}^{0}(p, q ; r, s)$ is independent of $\Lambda$. Since by dimensions it is dimensionless, by gauge invariance orthogonal to $p^{\mu}, q^{\nu}, r^{\lambda}$ and $s^{\sigma}$, and by locality polynomial in these momenta, the only solution is $S_{\mu \nu, \lambda \sigma}^{0}(p, q ; r, s)=0$. Clearly in this way one may establish that $S_{\mu \nu, \lambda_{1} \cdots \lambda_{n}}^{0}\left(p, q ; k_{1}, \cdots, k_{n}\right)$ 
vanishes for any number $n$ of gauge fields inside the second trace. (This remark is of importance for the large $N$ limit of the flow equation, as already mentioned in sect. 5.) The first non-vanishing double-trace vertex appears at the six-point level, with three gauge fields inside each trace.

Incidentally, we can now use (8.8) to confirm the statement above (4.17), that apart from $\operatorname{tr} \int d^{D} x F_{\mu \nu}^{2}$ there are no relevant or marginal operators here (as expected). We see by expanding (8.8) into $n$-point vertices and using $(8.13)$, that the term $\mathcal{O}_{\min }$ in $\mathcal{O}_{0}[A]$, with the lowest number $m_{\text {in }}$ of gauge fields, cannot have any $\Lambda$ dependence. Thus the (eigen)dimension of $\mathcal{O}$ (which about the Gaussian fixed point $g=0$, is that of $\mathcal{O}_{0}$ ) is simply $d_{\mathcal{O}}=m_{i n}+m_{p}$, where $m_{p}$ is the power of momentum in $\mathcal{O}_{\text {min }}$ (which must be a positive integer by quasilocality). Taking into account the requirement of gauge invariance we have that apart from the $m_{i n}=m_{p}=0$ unit operator (a.k.a. vacuum energy) which we here always ignore, and the $m_{i n}=m_{p}=2$ part of $\operatorname{tr} \int d^{D} x F_{\mu \nu}^{2}$ which by (4.16) amounts to a change of $g$, there are no operators with $d_{\mathcal{O}} \leq 4$.

\subsection{The $\beta$ function}

As usual, the $\beta$ function is determined through a renormalisation condition, which in our case is (4.16). Using this we have

$$
S_{\mu \nu}(p)=2 / g^{2} \Delta_{\mu \nu}(p)+O\left(p^{3}\right),
$$

and thus by $(8.13)$,

$$
S_{\mu \nu}(p)=\frac{1}{g^{2}} S_{\mu \nu}^{0}(p)+O\left(p^{3}\right)
$$

By (8.2), this implies that the $O\left(p^{2}\right)$ component of all the higher loop contributions $S_{\mu \nu}^{n}(p)$, must vanish. This greatly simplifies the $O\left(p^{2}\right)$ part of the two-point vertex flow in (8.5) (8.6), in particular reducing them to algebraic equations. Thus we see that

$$
a_{1}\left[S_{0}-2 \hat{S}\right]_{\mu \nu}(p)=-4 \beta_{1} \Delta_{\mu \nu}(p)+O\left(p^{3}\right)
$$

where $a_{1}\left[S_{0}-2 \hat{S}\right]_{\mu \nu}(p)$ is the two-point vertex in $a_{1}\left[S_{0}-2 \hat{S}\right]$. This fixes $\beta_{1}$. Similarly, at $n \geq 2$ loops, the $\beta_{n}$ are determined by the requirement that $a_{1}\left[S_{n-1}\right]_{\mu \nu}(p)=-4 \beta_{n} \Delta_{\mu \nu}(p)+$ $O\left(p^{3}\right)$. And non-perturbatively from (8.1) and (8.2),

$$
a_{1}\left[g^{2} S-2 \hat{S}\right]_{\mu \nu}(p)=-\frac{4}{g^{3}} \beta(g) \Delta_{\mu \nu}(p)+O\left(p^{3}\right)
$$




\subsection{One loop}

We can use the above classical vertices to compute the one-loop two-point vertex and thus $\beta_{1}$. The relevant diagrams for the LHS of (8.18) 8 are displayed in fig. 14, where the circle stands for $\Sigma_{0}=S_{0}-2 \hat{S}$.

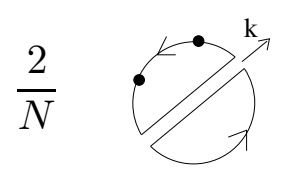

Fig.14. The one-loop two-point diagrams.

Translating the Feynman rules, we get

$$
\begin{array}{r}
a_{1}\left[\Sigma_{0}\right]_{\mu \nu}(p)=\frac{1}{\Lambda^{2}} \int \frac{d^{D} k}{(2 \pi)^{D}}\left\{c_{k}^{\prime} \Sigma_{\alpha \mu \nu \alpha}^{0}(k, p,-p,-k)+c_{\mu}^{\prime}(p ; k-p,-k) \Sigma_{\alpha \nu \alpha}^{0}(k,-p, p-k)\right. \\
\left.+\Sigma_{\alpha \alpha}^{0}(k) c_{\mu \nu}^{\prime}(p,-p ; k,-k)-\frac{c_{k}^{\prime}}{N^{2}}\left[\Sigma_{\alpha \mu \nu \alpha}^{0}(k, p,-p,-k)+\frac{1}{2} \Sigma_{\alpha \mu \alpha \nu}^{0}(k, p,-k,-p)\right]\right\} \\
+\left(p^{\mu} \leftrightarrow-p^{\nu}\right)
\end{array}
$$

Clearly by the Lorentz symmetry of the integral, the addition of the cyclic permutation $p^{\mu} \leftrightarrow-p^{\nu}$ just multiplies the result by two. Untying the large- $N$ change of variables $g \mapsto g / \sqrt{N}$, we see that $\beta_{1}$ in principle has $N$ dependence in two terms, one proportional to $N$, as expected, and one unexpected contribution proportional to $1 / N .9$ Actually this $1 / N$ term vanishes independently of the choice of covariantization. This follows since by using (5.3), Lorentz invariance $(k \leftrightarrow-k, \mu \leftrightarrow \nu$ etc. ) and the last (coincident line) identity in (7.4), one may show that under the $k$-integral $\hat{S}_{\alpha \mu \alpha \nu}(k, p,-k,-p) \equiv$ $-2 \hat{S}_{\alpha \mu \nu \alpha}(k, p,-p,-k)$, while expanding the $S_{0}$ four-point vertex by (8.16), and again using these symmetries, one may show that the same identity holds for $S_{\alpha \mu \alpha \nu}^{0}(k, p,-k,-p)$.

The problem is that the $k$ integral is divergent. While propagator-like terms have been UV improved to $\sim c / p^{2}$, the interactions e.g. (5.3), (8.14) and (8.16), are worse by $c^{-1}$, this latter behaviour being forced by gauge invariance (7.1), as we show in appendix A [3]. With a power-law regulator, i.e. $c(x) \sim 1 / x^{r}, r>0$, for large $x \equiv k^{2} / \Lambda^{2}$, the integrand $\sim 1 / k^{2}$ for large $k$. This behaviour may be established by analysing the individual contributions,

8 corresponding to $\Lambda \partial S_{\mu \nu}^{1}(p) / \partial \Lambda-2 \beta_{1} \hat{S}_{\mu \nu}(p)$, by (8.13) and (8.5).

9 The coefficient of each power of $N$ in $(8.19)$ is separately completely constrained by gauge invariance, up to an overall factor, in the sense outlined below (8.16). 
using the large $k$ behaviours given in appendix A. It is most readily seen to hold in the $a_{1}[\hat{S}]_{\mu \nu}(p)$ contributions: The $n$-point $\hat{S}$ vertices with two large momenta $\sim \pm k$ behave as $c_{k}^{-1} k^{4-n}$, while the $m$-point $c^{\prime}$ vertices with $\sim \pm k$ entering in at their 'ends' [as in (8.19)] behave as $c_{k}^{\prime} k^{-m}$. For the two-point one-loop vertex, we have $m+n=4$.

On taking into account the two powers of external momenta that must fall out by gauge invariance, as in (8.18), one might expect that the integrands behaviour is improved to $\sim 1 / k^{4}$, however this is false. Firstly, this power counting argument relies on dimensional reasoning which fails to work so simply here because it does not constrain functions of the dimensionless ratios $\sim$ momentum $/ \Lambda$. Secondly there is no separation of tree-level and loop contributions. These points are illustrated below. Finally, gauge non-invariant terms may also arise, from contributions that cancel, but only after a shift in $k$ [3]. These contributions thus integrate to surface terms in (8.19), which are guaranteed to vanish only if the integral over the original parts converge.

To demonstrate this effect we compute the gauge dependent part of (8.19). The momentum routing chosen in fig. 14 and (8.19) corresponds to taking $k$ to be the momentum of the left-most functional derivative in the quantum term of (4.18) and is natural if we wish to further regularise the integral in a way that corresponds to modifying the measure over $A_{\mu}(k)$ modes whilst preserving the fact that (4.18) leaves the partition function (3.4) invariant, and thus preserve the expected universality of $\beta_{1}$. However the reader can check that $k$-shifted differences always arise, but as differing expressions, no matter how the terms in (8.19) are routed. (This reflects the conflict between specifying momenta, and the action of gauge invariance [18] [3].) We assume that (8.19) is further regularised so that invariance under $k \leftrightarrow-k$ is preserved, and collect terms using the observation above (7.3). Then using (7.1), (7.2), and (8.13), we readily obtain

$$
p^{\mu} p^{\nu} a_{1}\left[\Sigma_{0}\right]_{\mu \nu}(p)=\frac{2}{\Lambda^{2}} \int \frac{d^{D} k}{(2 \pi)^{D}}\left\{c_{k}^{\prime} \hat{S}_{\alpha \alpha}(k)-c_{k+p}^{\prime} \hat{S}_{\alpha \alpha}(k+p)\right\} .
$$

Using (5.3) and expanding the RHS as a power series in $p$, one obtains integrals that may be done exactly and thus, using (8.18),

$$
\begin{aligned}
& a_{1}\left[\Sigma_{0}\right]_{\mu \nu}(p)=-4(D-1) \Omega_{D} \Lambda^{D-4}\left\{\Lambda^{2} \delta_{\mu \nu}\left[G_{0}\right]_{0}^{\infty}+p_{\mu} p_{\nu}\left[G_{L}\right]_{0}^{\infty}\right\}-4 \beta_{1} \Delta_{\mu \nu}(p)+O\left(p^{3}\right) \\
& \text { where } \quad G_{0}=\frac{1}{D} x^{D / 2}\left(x c^{\prime} / c\right)^{\prime} \quad \text { and } \quad G_{L}=\frac{1}{D(D+2)}\left[x^{D / 2+1}\left(x c^{\prime} / c\right)^{\prime \prime}\right]^{\prime}
\end{aligned}
$$

Here $\Omega_{D}=2 /\left[\Gamma(D / 2)(4 \pi)^{D / 2}\right]$ is the solid angle of a $(D-1)$-sphere divided by $(2 \pi)^{D}$, and as before prime is differentiation with respect to its argument (here $x$ ). These expressions 
give some guide as to the typical divergences expected using the cutoff function $c(x)$. In particular for the power-law $c$, we have

$$
x c^{\prime} / c \rightarrow-r \quad \text { as } \quad x \rightarrow \infty \quad
$$

and the leading divergences actually cancel. If in addition the correction to (8.22) is $O(1 / x)$ or better (as will be the case here), the non-invariant terms in (8.21) vanish for all $D<4$, and thus also for $D \rightarrow 4^{-}$. Note that at $D=4$, we could still get a finite $O\left(p^{0}\right)$ term from the subleading correction to (8.22), violating gauge invariance. This illustrates the need to define these integrals carefully. As we will see below however, power-law $c$ is not enough to regularise the gauge-invariant divergences.

Consider fig. 15. This is one of several $\sim 1 / k^{2}$ contributions to the first term of (8.19) (from expanding the first term in fig. 12, using fig. 11) which, since the two blobs appear on two-point $\hat{S}$ vertices, are already orthogonal to $p$. Another example is given in ref. [3].

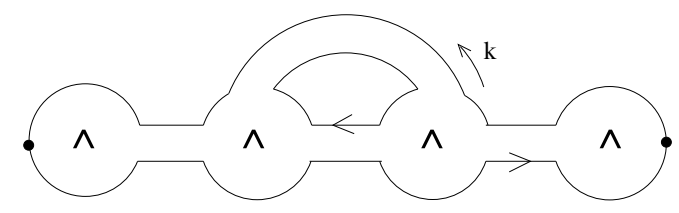

Fig.15. Divergent one-loop contribution (made one-loop by the curved wine).

These outer two-point vertices do not feel the loop-momentum $k$, nor indeed do the outer wines, and in this sense are really tree corrections to the one-loop term. Indeed, however many points appear on these outer parts the divergence is still quadratic in $D=$ 4 (i.e. integrand goes as $\sim 1 / k^{2}$ ). Note that the two outer wines external momentum dependence $c_{p}^{\prime}$ ensure that the coefficient of this divergence is even non-polynomial of the form

$$
\int_{\Lambda}^{\infty} \frac{d \Lambda_{1}}{\Lambda_{1}^{3}} \cdots\left(\int_{\Lambda_{1}}^{\infty} \frac{d \Lambda_{2}}{\Lambda_{2}^{3}} c_{p}^{\prime} c_{p}^{-1} \cdots\right)^{2}
$$

(The non-polynomial part arises from the $c_{p}^{\prime}$ and it should be understood that the inner $c$ 's are functions of $\Lambda_{2}$.) It is easy to verify either diagrammatically or directly from (8.14), (8.16) and (8.19), that no other contribution has the same non-polynomial dependence, and thus this divergence remains uncancelled. Using appendix A, we isolated exactly the complete coefficients (polynomial and non-polynomial) of the leading (and sub-leading) divergences, and verified that there are many further uncancelled divergences. In the interest of compactness we do not present the details. 


\section{Conclusions}

We briefly recapitulate some of the main points.

We formulate a flow equation for the effective action $S$ of a non-Abelian gauge field $A_{\mu}$, the connection for the covariant derivative $D_{\mu}=\partial_{\mu}-i A_{\mu}$, which leaves the partition function invariant under the flow.

Gauge invariance is manifest at all stages; the transverse bilinear term in $S$ need not be inverted in the equation or its solution (which is here investigated perturbatively). Ghosts and gauge fixing are not required. The challenging problem of Gribov copies is thus avoided.

(N.B. Contrary to popular folklore, it is not necessary to implement gauge fixing or other explicit reduction of variables merely to factor out the infinite volume of the gauge group, which in any case only amounts to one of many harmless divergent vacuum energy contributions.)

The placing of the coupling constant $g$ outside the connection, together with the exact preservation of gauge invariance, means that $A_{\mu}$ is protected from wavefunction renormalization. Only $g$ renormalizes. We show how the corresponding $\beta$ function is determined through the appropriate renormalization condition on $S$.

The global group invariance ensures that everything can be expanded in products of traces. We show that in the large $N$ limit, $S$ collapses to a single trace. The limit of the flow equation itself may be more subtle.

The general covariantization entering into the equation can be conveniently expressed as an integral over configurations of Wilson lines. In a similar way the solution $S$ may be expressed as an integral over a 'gas' of Wilson loops. In this way the flow equation may be reformulated entirely in terms of manipulations on Wilson loops. The equations then determine the measure over these fluctuating Wilson loops. In the large $N$ limit, $S$ becomes an integral over the configurations of just one Wilson loop, i.e. a path integral for a single particle circulating in a loop.

This Wilson loop picture may also be interpreted as Feynman diagrams and thus provides an intuitive and elegant way to derive the perturbative solution for $S$. We derive the 'Ward identities' and discuss the interpretation of the various symmetries (charge conjugation, Lorentz and gauge invariance) in this picture. We derive the two, three and four point vertices at the classical level, and the one-loop two-point vertex.

We explicitly confirm that these solutions are gauge invariant. However, we show that subtleties with momentum-integral surface terms appear at one loop (and higher) as a 
consequence of the divergent nature the momentum integrals in this framework. This in turn is related to the large momentum behaviour of the vertices, whose lower bound is fixed by the exact preservation of gauge invariance (as we show in appendix A). We demonstrate that some of the divergences that thus appear at one loop are non-polynomial in momenta and remain uncancelled in the complete solution in this framework. The further regularisation that is required is developed in ref. [9].

The flow equation and its solution $S$ enjoy the important property of 'quasilocality', namely that they can be expanded in a Taylor series in their (external) momentum arguments to all orders. Equivalently for $S$, that it has a derivative expansion to all orders. The completely regularised flow equation will correspond to integrating out high energy modes because $\Lambda$ will then act properly as an effective ultraviolet cutoff. The preservation of the partition function then ensures that those high energy modes that are eliminated, get incorporated into $S$. The solution to the flow equation thus yields for the first time, a precise continuum prescription for a gauge invariant Wilsonian effective action.

\section{Acknowledgements}

The author acknowledges support of the PPARC through an Advanced Fellowship, and grant GR/K55738.

\section{Appendix A. Large and small momenta}

Any vertex has a transverse part whose form depends on the choice of covariantization

\{\} (and the choice of flow equation in the case of $S$ ), and a universal longitudinal part whose form is dictated by gauge invariance. Consider the one-point wine $W_{\mu}(p ; k,-k-p)$. (Recall that we label the one-point vertex by the kernel it covariantizes. Since, by translation invariance, all vertices are accompanied by momentum conserving delta functions, all relations must be derived for the case where the momentum arguments sum to zero.) By gauge invariance (7.1), (7.2), we have (7.4):

$$
p^{\mu} W_{\mu}(p ; k,-k-p)=W_{k}-W_{k+p} .
$$


In the case that $p$ and $k$ are colinear, this may be solved immediately for $W_{\mu}(p ; k,-k-p)$. More generally, for small $p$, using $W_{k} \equiv W\left(k^{2} / \Lambda^{2}\right)$, we have

$$
W_{\mu}(p ; k,-k-p)=-\frac{W_{k}^{\prime}}{\Lambda^{2}}\left(2 k_{\mu}+p_{\mu}\right)-2 \frac{W_{k}^{\prime \prime}}{\Lambda^{4}} k \cdot p k_{\mu}+O\left(p^{2}\right)
$$

where we have noted that any term perpendicular to $p^{\mu}$ may be added while leaving (A.1) unchanged, but by Lorentz invariance this has tensor structure $\Delta_{\mu \nu}(p) k^{\nu}$.

(A.1) also constrains the ultraviolet behaviour of $W_{\mu}(p ; k,-k-p)$. For example, for large $k$ it is constrained to be at least as bad as the RHS. Explicitly, consider choices of $W_{k}$ where the scale is set by $k$ for large momenta, and thus $W^{\prime}(x) / W(x) \sim 1 / x$ for large $x$, i.e. where $W_{k}$ goes as a power or log for large $k$ (say $W(x) \sim x^{w}$ ). (A.2) then typically holds also for $k \gg p$, with $O\left(p^{2}\right)$ replaced by $O\left(p^{2} k^{2 w-3}\right)$, since for this to be violated requires including non-minimal field strength terms 10 in \{\} .

Many similar relations can be derived for colinear, small and large momenta for the other vertices, including $\hat{S}$ and $S$ vertices. In any case we have that the ultraviolet behaviour for large $k$ is at best $W_{\mu}(p ; k,-k-p) \sim W_{k} / k$. It is then easy to see that $W_{\mu_{1} \cdots \mu_{n}, \nu_{1} \cdots \nu_{m}}\left(p_{1}, \cdots, p_{n} ; q_{1}, \cdots, q_{m} ; k,-k-\sum_{i} p_{i}-\sum_{j} q_{j}\right)$ must have ultraviolet behaviour at least as bad as $W_{k} / k^{m+n}$ : By the gauge relations (7.1), (7.2), each $m+n$ vertex is related to a difference of two $(m+n-1)$ vertices, whose arguments only differ in momenta small with respect to $k$. For choices $W_{k}$ where $k$ sets the scale (as above), this difference falls by just one extra power of $k$ compared to each $(m+n-1)$ vertex, and thus the $m+n$ vertex cannot fall faster than an $(m+n-1$ vertex $) / k$. The italicized statement above then follows immediately, by iteration.

Recall that the two-point vertices for the actions are fixed as in (8.13) and (5.3), and therefore $S_{\mu \nu}(k)=\hat{S}_{\mu \nu}(k) \sim c_{k}^{-1} k^{2}$. By the same argument as above, using (7.1), we have that the $n$-point vertices $\hat{S}_{\mu_{1} \cdots \mu_{n}}\left(p_{1}, \cdots, p_{n}\right)$ where any two momenta $p_{i}$ are large of order $k$, behave at best as $c_{k}^{-1} k^{4-n}$. The same bound holds for $S$. These relations are sufficient for the demonstration that the one-loop contribution is not regularized (in sect. 8), and show that the lack of complete regularization follows from the gauge invariance.

In fact, while the $\hat{S}$ vertices achieve the lower bound $c_{k}^{-1} k^{4-n}$ (as sketched below), $S$ does not, as is already clear from the flow of the classical four-point function shown in fig. 12. We see there that if the two large momenta are next to each other, they can enter and leave a three point lobe, thus actually $S_{\mu \nu \lambda \sigma}(k,-k-p-q, p, q) \sim c_{k}^{-1} k$.

10 balanced by $1 / \Lambda^{2}$ : these are the terms which are transverse to $p$ 
This effect would not appear if the gauge invariant flow was for one-particle irreducible vertices (a Legendre formulation) but the lack of separation between tree-level and loop contributions (see also the end of sect. 8) seems to be a necessary consequence of the exact preservation of gauge invariance. To see this, recall that all the terms in fig. 12 have to appear as shown, by gauge invariance, given the first two terms. But attaching a wine to make a one-loop contribution out of this gauge invariant four-point vertex produces both diagrams that are one-particle reducible and one-particle irreducible.

The cases where one or both large momenta enter the side of a wine are also more involved. We rewrite (A.1) as

$$
k^{\mu} W_{\mu}(k ; p,-k-p)=W_{p}-W_{k+p}
$$

Now the asymptotic behaviour for large $k$ depends on whether $W_{k}$ grows or falls for large $k$. The growing case corresponds to $c_{k}^{-1} \sim k^{2 r}$ and does still follow the simple rules above, if $r$ is large enough: We have that at best $c_{\mu}^{-1}(k ; p,-k-p) \sim c_{k}^{-1} / k$. Arguments as above then rapidly establish that an $m+n$ point vertex from $\left\{c^{-1}\right\}$ with two large momenta $\sim k$ in any position behaves at best as $\sim c_{k}^{-1} / k^{m+n}$ providing $r \geq m+n$. And once again, this bound is saturated if there are no non-minimal terms in \{\} . In the cases where $r<m+n$, the bound established below for the falling cases may dominate.

The falling case corresponds to $W_{k}=c_{k}^{\prime}$ and in this case we read from (A.3), that at best $c_{\mu}^{\prime}(k ; p,-k-p) \sim c_{p}^{\prime} / k$. Note that the coefficient of a divergence arising from this need not be polynomial in $p$. (See similar remarks in sect. 8.) This $1 / k$ behaviour is true of any $m+n$ vertex where the $k^{\mu}$ can reach the top end of the line, for example:

$k^{\mu} c_{\lambda \mu, \nu}^{\prime}(r, k ; q ; p,-k-p-q-r)=c_{\lambda, \nu}^{\prime}(r ; q ; p,-p-q-r)-c_{\lambda, \nu}^{\prime}(r+k ; q ; p,-k-p-q-r)$

The first term on the RHS $\sim k^{0}$ sets the bound, since the second term can go as $\sim$ $1 / k$ [as may be established by writing out the gauge relation $k^{\lambda} c_{\lambda, \nu}^{\prime}(k ; q ; p,-k-p-q)$ ]. Again by iteration, the general rule for the case where one large momentum $k$ enters via the side of $\left\{c^{\prime}\right\}$ is that the vertex goes at best as $1 / k^{v+1}$ where $v$ is the number points (gauge fields) separating the two large momenta. Once more, this is saturated by minimal covariantizations.

The case where both large momenta enter $\left\{c^{\prime}\right\}$ via the side can be treated similarly, with the conclusion that the vertex again goes at best as $1 / k^{v+1}$ where $v$ is the number points separating the two large momenta. However in this case even minimal covariantizations 
do not necessarily reach this bound. For (4.13), the case where the two momenta are next to each other on one side of the wine leads to a $O\left(k^{0}\right)$ contribution $11 \sim \Delta_{\mu \nu}(k) / k^{2}$ as will be established explicitly in ref. [9]. Consequently (4.13) actually behaves as $1 / k^{v}$, for two large momenta $\sim k$ entering the side and separated by $v$ points.

As indicated above if $r<m+n$, in particular if $m+n-r>v+1(m+n-r>v)$, then the bounds $\sim 1 / k^{v+1}\left(\sim 1 / k^{v}\right)$ for one (two) large side momenta are the limiting ones for $\left\{c^{-1}\right\}$ also. With the above bounds for wine vertices, one can check explicitly from (5.3), that the $\hat{S}$ vertices saturate their own bounds. All the asymptotic formulae given in this appendix will be confirmed explicitly for (4.13) in ref. [9], where we will also derive the coefficient functions multipying the leading $k$ behaviour.

11 Note that it is necessarily transverse, otherwise the $k^{\mu}$ gauge relations would be violated. 


\section{References}

[1] K. Wilson and J. Kogut, Phys. Rep. 12C (1974) 75.

[2] D.U. Jungnickel and C. Wetterich, in The Exact Renormalization Group, Eds Krasnitz et al, World Sci (1999) 41, and hep-ph/9902316.

[3] T.R. Morris, in The Exact Renormalization Group, Eds Krasnitz et al, World Sci (1999) 1, and hep-th/9810104 (this is updated to conform to present notation).

[4] T.R. Morris, in Yukawa International Seminar '97, Prog. Theor. Phys. Suppl. 131 (1998) 395, hep-th/9802039.

[5] T.R. Morris, in New Developments in Quantum Field Theory, NATO ASI series 366, (Plenum Press, 1998), hep-th/9709100.

[6] T.R. Morris, in RG96, Int. J. Mod. Phys. B12 (1998) 1343, hep-th/9610012.

[7] See for example the reviews: K. Intriligator and N. Seiberg, Nucl. Phys. Proc. Suppl. 45BC (1996) 1, hep-th/9509066; C. Gomez and R. Hernandez, hep-th/9510023; L. Alvarez-Gaumé and S.F. Hassan, Fortsch. Phys. 45 (1997) 159, hep-th/9701069.

[8] S. Arnone, C. Fusi and K. Yoshida, J. High Energy Physics 02 (1999) 022.

[9] T.R. Morris, part II in preparation

[10] G. 't Hooft, Nucl. Phys. B72 (1974) 461.

[11] A. Migdal, Ann. Phys. 109 (1977) 365; Yu.M. Makeenko and A.A. Migdal, Nucl. Phys. B188 (1981) 269.

[12] A.A. Migdal, Phys. Rep. 102 (1983) 199.

[13] A.M. Polyakov, Gauge fields and Strings (Harwood, 1987).

[14] V.A. Kazakov and I.K. Kostov, Nucl. Phys. B176 (1980) 199; V.A. Kazakov, Nucl. Phys. B179 (1981) 283.

[15] See the review by A.V. Manohar, in PANIC 96, hep-ph/9607484; M.J. Teper, heplat/9804008.

[16] A.A. Slavnov, Theor. Math. Phys. 13 (1972) 1064; B.W. Lee and J. Zinn-Justin, Phys. Rev. D5 (1972) 3121.

[17] V. Gribov, Nucl. Phys. B139 (1978) 1; I. Singer, Comm. Math. Phys. 60 (1978) 7; See e.g. C. Becchi, hep-th/9607181; P. van Baal, hep-th/9711070; M. Asorey and F. Falceto, Ann. Phys. 196 (1989) 209; K. Fujikawa, Nucl. Phys. B468 (1996) 355.

[18] M. D'Attanasio and T.R. Morris, Phys. Lett. B378 (1996) 213.

[19] C. Becchi, in Elementary Particles, Field Theory and Statistical Mechanics, (Parma, 1993), hep-th/9607188; M. Bonini et al, Nucl. Phys. B409 (1993) 441, B418 (1994) 81, B421 (1994) 81, B437 (1995) 163; U. Ellwanger, Phys. Lett. 335B (1994) 364; U. Ellwanger et al, Z. Phys. C69 (1996) 687; M. Reuter and C. Wetterich, Nucl. Phys. B417 (1994) 181, B427 (1994) 291; K-I. Aoki et al, Prog. Theor. Phys. 97 (1997) 479, hep-th/9908042, hep-th/9908043; K-I. Kubota and H. Terao, hep-th/9908062; 
M. Pernici et al, Nucl. Phys. B520 (1998) 469; D.F. Litim and J.M. Pawlowski, Phys. Lett. B435 (1998) 181; M. Simionato, hep-th/9809004.

[20] J. Polchinski, Nucl. Phys. B231 (1984) 269.

[21] F.J. Wegner and A. Houghton, Phys. Rev. A8 (1973) 401.

[22] J. Zinn-Justin, "Quantum Field Theory and Critical Phenomena" (1993) Clarendon Press, Oxford.

[23] T.R. Morris, Int. J. Mod. Phys. A9 (1994) 2411.

[24] M. Salmhofer, Nucl. Phys. B (Proc. Suppl.) 30 (1993), 81.; C. Wetterich, Phys. Lett. B301 (1993), 90; M. Bonini et al, Nucl. Phys. B418 (1994), 81.

[25] T.R. Morris, Phys. Lett. B357 (1995) 225.

[26] C. Becchi, A. Rouet and R. Stora, Commun. Math. Phys. 42 (1975) 127.

[27] T.R. Morris, Nucl. Phys. B458[FS] (1996) 477. 